\title{
A comparative study of the vibro-impact capsule systems with one-sided and two-sided constraints
}

\author{
Yao Yan · Yang Liu $\mathbb{D}$ - Maolin Liao
}

Received: 30 December 2016 / Accepted: 24 March 2017 / Published online: 7 April 2017

(C) The Author(s) 2017. This article is an open access publication

\begin{abstract}
This paper studies the dynamics of the vibro-impact capsule systems with one-sided and twosided soft constraints under variations of various system and control parameters, including mass ratio, stiffness ratio, gap of contact, and amplitude and frequency of external excitation. The aim of this study is to optimise the progression speed and energy consumption of the capsule and minimise the required cabin length for prototype design used for engineering pipeline inspection. Our studies focus on three systems: the capsule with a right constraint, the capsule with a right and a weak left constraints, and the capsule with a right and a strong left constraints. Bifurcation analyses show that the behaviour of the capsule with onesided constraint is mainly periodic, and the dynamic responses of the other two capsules with two-sided constraints become complex when the stiffness of the left constraint increases. Based on our extensive comparisons, the following optimisation strategies are recommended. When the capsule speed is paramount, one
\end{abstract}

Y. Yan

School of Aeronautics and Astronautics, University of Electronic Science and Technology of China, Chengdu 611731, China

Y. Liu $(\bowtie)$

College of Engineering Mathematics and Physical

Sciences, University of Exeter, Rennes Drive,

Exeter EX4 4RN, UK

e-mail: y.liu2@exeter.ac.uk

M. Liao

CNPC Drilling Research Institute, Beijing 102206, China can employ the two-sided capsule with a weak left constraint under large amplitude of excitation. When energy consumption is taken into account, the onesided capsule is preferable. When a miniaturized prototype is needed, the two-sided capsule with a strong left constraint is the best choice.

Keywords Vibro-impact · Non-smooth dynamical system · Stick-slip · Optimisation · Energy consumption

\section{Introduction}

Pipelines play an important role in a large number of modern industries [1,2], transporting fluids from one location to another, whether from one side of a factory to the other or across the breadth of entire continents. They are essential assets within water supplies, oil and gas production, and many other vital industries throughout the globe. Pipelines vary greatly in diameter, length, construction material, and location. With many pipelines being located in remote and harsh locations, such as being located underground or running along the seabed, access for inspection, maintenance and repair work could be extremely difficult. The challenges also include accurately locating leaks or blockages within operational pipelines, and monitoring corrosion or deterioration on internal surfaces. It becomes a particularly costly issue if the pipeline has to be drained and production stopped while repair work takes 
place. Therefore, pipeline inspection devices capable of moving independently, with or against product flow would yield significant advantages over traditional pressure driven inspection tool [3] in certain situations. In recent years, investigation of the selfpropelled mechanism moving rectilinearly under internal vibration force when overcoming medium resistance has attracted great attention from researchers, e.g. [4-8]. The principle of such mechanism is that the rectilinear motion can be obtained by overcoming external resistance described as dry friction using an additional internal mass interacting with the main body of the system. The ability to move independently without any external moving parts makes it ideally suited to move in harsh and complex environments, where external moving parts may either pose a hazard to the surroundings or where they would be likely to be broken, corroded, or blocked up by the working environment.

This paper studies the optimisation of the vibroimpact capsule systems [8-10] with one-sided and twosided amplitude constraints for engineering pipeline inspection in terms of its average speed of progression, physical dimension, and energy consumption. Optimisation of motion of the self-propelled mechanism regarding to average speed of progression has been an active subject of scientific research. For example, Chernousko [4] pioneeringly studied the optimum rectilinear motion of a two-mass system and obtained the optimum control parameters for the maximum mean velocity of the system. In [6,11], optimisation of a mobile system with an internal acceleration-controlled mass was considered to obtain the maximum forward mean speed. Later on, Fang and Xu [12] studied the dynamics of a multibody system consisting of three modules of such vibration-driven mobile system. Considering the controlled motion a rigid body in the horizontal plane, Zhan and $\mathrm{Xu}$ [13] used three internal acceleration-controlled masses to drive the system. Recently, optimisation of two-dimensional motion of the vibration-driven system has been extensively studied, e.g. [14,15]. However, these studies focused on theoretical calculation of the maximum mean speed, and few works concerned optimisation of the mobile system from a practical design point of view, such as physical dimension and energy consumption. Vetchanin et al. [16] investigated the characteristics of motion of a rigid body with variable internal mass distribution in a viscous fluid, showing the possibility of self-propulsion of the body in an arbitrary given direction. In [17], exper- imental verification of the vibro-impact capsule system was carried out. The conducted bifurcation analyses indicated that a fine tuning of the control parameters, such as the stiffness of the support spring, and the frequency and the amplitude of excitation, can significantly improve the average rate of progression. In [18], optimisation of the vibro-impact capsule system for the best progression in fluid environment was studied, focusing on the choice of the excitation parameters and the shape of the capsule. This paper will further study the optimisation of the vibro-impact capsule system regarding to its physical dimension and energy consumption through bifurcation analysis. Three capsule dynamics with one-sided and two-sided amplitude constraints will be compared in order to obtain the best design parameters for prototyping.

The dynamics of the vibro-impact capsule system, which consists of a capsule main body interacting with a harmonically driven internal mass, has been studied extensively by Liu et al. [8-10,17-20]. In [8], the model of the vibro-impact capsule system was firstly studied to provide a fundamental understanding of its dynamics. Dynamics of the system in various frictional environments was investigated in [9], and numerical results show that the behaviour of the system becomes very complex when the capsule is moving in fluid, but the nature of the friction mechanism becomes less significant when the weight of the internal mass is smaller than the weight of the capsule. In [10], nonlinear dynamics analysis has been conducted to identify the optimal amplitude and frequency of the applied force to achieve the required motion and the maximal speed. In [19], Páez Chávez et al. studied two practical problems for the capsule system, which were maximising the rate of progression and directional control of the system by following a typical period-1 trajectory by means of path-following techniques. However, the above studies were based on the dynamics of the capsule system with one-sided constraint, and the performance of the system with two-sided constraints has not been investigated. Thus, the contribution of this paper is to study the capsule system with two-sided soft constraints and understand how does the additional constraint affect the performance of the capsule. There are some existing studies on the vibro-impact systems with two-sided constraints, but most of them focused on the systems with bilateral rigid constraints. For example, Gutiérrez and Arrowsmith [21] considered a representative model of the doubly constrained impacting system, and studied 
Fig. 1 Physical models of the vibro-impact capsule systems, with a double-sided constraints, and $\mathbf{b}$ one-sided constraint. c Free body diagrams of the inner mass $m_{1}$ and the capsule $m_{2}$ (a)

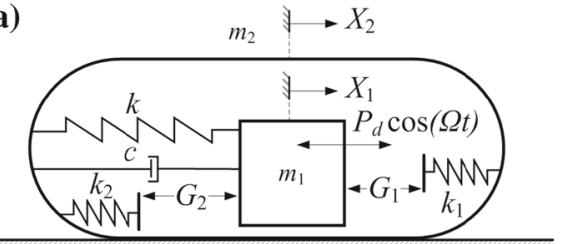

(b)

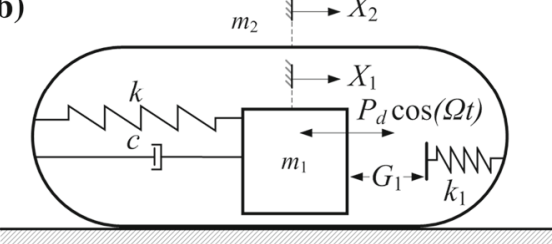

(c)

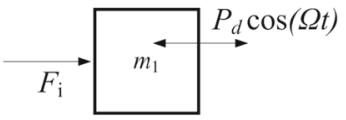

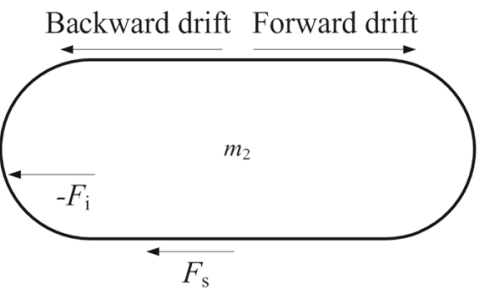

the control strategies for preservation and annihilation of experimental and analytical resonant periodic orbits. Lee and Yan [22] developed a position control method for the impact oscillator with asymmetrical doublesided endstops, which can keep the stable and the unstable oscillators in a desired position. Luo et al. [23] studied the vibro-impact dynamics of a two-degreeof-freedom periodically forced system with a bilateral clearance. Response analysis for a vibro-impact Duffing system with bilateral barriers under external and parametric Gaussian white noises was carried out by Yang et al. [24]. Very recently, Kumar et al. [25] analysed a stochastically excited vibro-impact Duffing-Van der Pol oscillator with bilateral rigid barriers. However, the literature on the vibro-impact systems with doublesided soft constraints is rather limited. Andreaus and Angelis [26] studied the dynamic response of a singledegree-of-freedom oscillator constrained by two unilateral nonlinear bumpers. Hao et al. [27] developed a twosided damping constraint control strategy for the quasizero stiffness isolator. The study by Ing [28] revealed that, in a near symmetrical system, the degree of asymmetry was found to have a vast effect on the response of a double-sided constraint impact oscillator. In this paper, we will investigate whether such asymmetry can be used for optimising the physical performance of the capsule system, e.g. progression rate, power efficiency, and capsule dimension. In practice, the stroke length of the internal mass, i.e. the maximum travel distance of the mass in one period of motion, constrains the physical dimension and power consumption of the capsule system, which in turn affects the performance of the system. Thus, it is reasonable to carry out a comparative study in this paper, which can provide a better insight for the design of such system with consideration of its physical performance, and this is the novelty of this paper.

The rest of this paper is organised as follows. In Sect. 2, mathematical modelling of the vibro-impact capsule systems with one-sided and two-sided constraints is studied. In Sect. 3, bifurcation analysis of the capsule system is conducted through varying various system and control parameters. Optimisation of energy consumption and cabin length are considered in Sect. 4, and finally, some concluding remarks are drawn in Sect. 5.

\section{Mathematical modelling}

\subsection{Description of the capsule system}

Consider a two degrees-of-freedom system as shown in Fig. 1a, which is composed of a movable internal mass $m_{1}$ interacting with a rigid capsule $m_{2}$ via a primary linear spring with stiffness $k$ and a viscous damper with damping coefficient $c$. The internal mass is driven by an external harmonic force with amplitude $P_{d}$ and frequency $\Omega$. In practice, this can be implemented using a linear actuator (e.g. solenoid [29]). It is worth noting that the interaction between the $\operatorname{rod}\left(m_{1}\right)$ and the main body $\left(m_{2}\right)$ of the actuator is approximated using a linear spring and a viscous damper in this paper, and the verification of such assumption was carried out in [17]. On the right of the internal mass, a weightless plate is connected to the capsule by a linear spring with stiffness $k_{1}$, and a secondary weightless plate is connected to the capsule by a linear spring with stiffness $k_{2}$ on 
the left of the internal mass. Here, $X_{1}$ and $X_{2}$ represent the absolute displacements of the internal mass and the capsule, respectively. The internal mass will contact with the right plate when the relative displacement $X_{1}-X_{2}$ is larger or equals to the gap $G_{1}$, or contact with the left plate when the relative displacement $X_{2}-X_{1}$ is larger or equals to the gap $G_{2}$. When the left spring is removed (i.e. $k_{2}=0$ ) as shown in Fig. $1 b$, the system has only one-sided constraint which has been thoroughly studied by Liu et al. [8-10].

\subsection{Equations of motion}

Due to the non-smoothness introduced by the gaps and the friction, equations of motion of the capsule system should be considered in different phases. For the non-smoothness of gaps $G_{1}$ and $G_{2}$, mutual interaction between $m_{1}$ and $m_{2}$ has three different cases: no contact $\left(X_{1}-G_{1}-X_{2}<0\right.$ and $\left.X_{2}-G_{2}-X_{1}<0\right)$, right contact $\left(X_{1}-G_{1}-X_{2} \geq 0\right)$ and left contact $\left(X_{2}-G_{2}-X_{1} \geq 0\right)$. Thus, the interactive force is given as

$F_{i}= \begin{cases}-c\left(\dot{X}_{1}-\dot{X}_{2}\right)-k\left(X_{1}-X_{2}\right), & \text { (no contact) } \\ -c\left(\dot{X}_{1}-\dot{X}_{2}\right)-k\left(X_{1}-X_{2}\right)-k_{1}\left(X_{1}-G_{1}-X_{2}\right), & \text { (right contact) } \\ -c\left(\dot{X}_{1}-\dot{X}_{2}\right)-k\left(X_{1}-X_{2}\right)-k_{2}\left(X_{1}+G_{2}-X_{2}\right), & \text { (left contact) }\end{cases}$

or written as

$$
\begin{aligned}
F_{i}= & -c\left(\dot{X}_{1}-\dot{X}_{2}\right)-k\left(X_{1}-X_{2}\right) \\
& -H_{1} k_{1}\left(X_{1}-G_{1}-X_{2}\right) \\
& -H_{2} k_{2}\left(X_{1}+G_{2}-X_{2}\right),
\end{aligned}
$$

where $H_{1}$ and $H_{2}$ are the Heaviside functions given by

$$
\begin{aligned}
& H_{1}=H\left(X_{1}-G_{1}-X_{2}\right), \\
& H_{2}=H\left(X_{2}-G_{2}-X_{1}\right) .
\end{aligned}
$$

The second non-smoothness of the system is introduced by the friction $F_{S}$ between the capsule and its supporting surface when the capsule moves horizontally $\left(\dot{X}_{2} \neq 0\right)$ as depicted in Fig. 1c. Here, the Coulomb friction model is used to describe the frictional force given by

$$
F_{s}=-\operatorname{sign}\left(\dot{X}_{2}\right) P_{f} \text {, }
$$

where $P_{f}=\mu\left(m_{1}+m_{2}\right) g, \mu$ is the friction coefficient between the capsule and the environmental surface, and $g$ is the acceleration due to gravity. When the capsule is stationary $\left(\dot{X}_{2}=0\right)$, two situations could happen. If the elastic force acting on the capsule is greater than the threshold of friction, i.e. $\left|F_{i}\right|>P_{f}$, the capsule begins to move and the direction of friction force is opposite to the elastic force. At this moment, the dry friction force is calculated as

$F_{s}=-\operatorname{sign}\left(F_{i}\right) P_{f}$,

When the force acting on the capsule from the internal mass is smaller than the threshold of friction, i.e. $\left|F_{i}\right| \leq P_{f}$, the friction force becomes static which is calculated as

$F_{s}=-F_{i}$.

Considering all the conditions above, the comprehensive friction force can be written as

$$
\begin{aligned}
F_{s}= & -\left(1-\Delta_{\mathrm{v}}\right) S_{v} P_{f}-\Delta_{\mathrm{v}} H_{f} S_{f} P_{f} \\
& -\Delta_{\mathrm{v}}\left(1-H_{f}\right) F_{i},
\end{aligned}
$$

where $\Delta_{\mathrm{v}}=\delta\left(\dot{X}_{2}\right)$ is the Dirac Delta function, $H_{f}=$ $H\left(\left|F_{i}\right|-P_{f}\right)$ is the Heaviside function, and $S_{v}=$ $\operatorname{sign}\left(\dot{X}_{2}\right)$ and $S_{f}=\operatorname{sign}\left(F_{i}\right)$ are the sign functions.

Based on the free diagram shown in Fig. 1c, the equations of motion of the capsule system can be written as

$$
\begin{aligned}
& m_{1} \ddot{X}_{1}(t)=P_{d} \cos (\Omega t)+F_{i}, \\
& m_{2} \ddot{X}_{2}(t)=F_{i}-F_{s} .
\end{aligned}
$$

For simplification, we introduce the following nondimensional parameters

$$
\begin{aligned}
\Omega_{0} & =\sqrt{\frac{k}{m_{1}}}, \quad \omega=\frac{\Omega}{\Omega_{0}}, \\
\alpha & =\frac{P_{d}}{P_{f}}, \quad \zeta=\frac{c}{2 m_{1} \Omega_{0}}, \\
\gamma & =\frac{m_{2}}{m_{1}}, \quad \delta_{1}=\frac{k}{P_{f}} G_{1}, \\
\delta_{2} & =\frac{k}{P_{f}} G_{2}, \quad \kappa_{1}=\frac{k_{1}}{k}, \\
\kappa_{2} & =\frac{k_{2}}{k},
\end{aligned}
$$


Fig. 2 Runge-Kutta method with a constant time step and $\mathbf{b}$ the bisection method for integration. The critical points to be located are marked by red triangles. (Color figure online) (a)

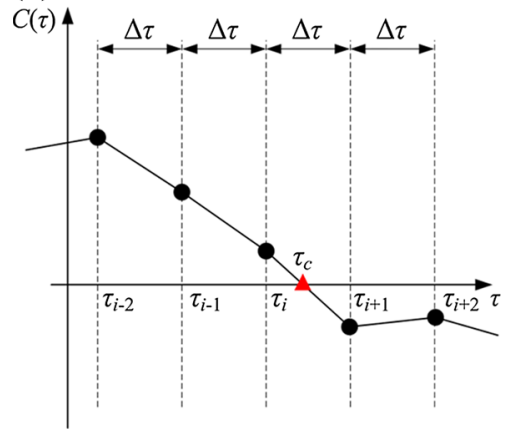

(b)

$\left|C\left(\tau^{n}\right)\right|<\varepsilon$

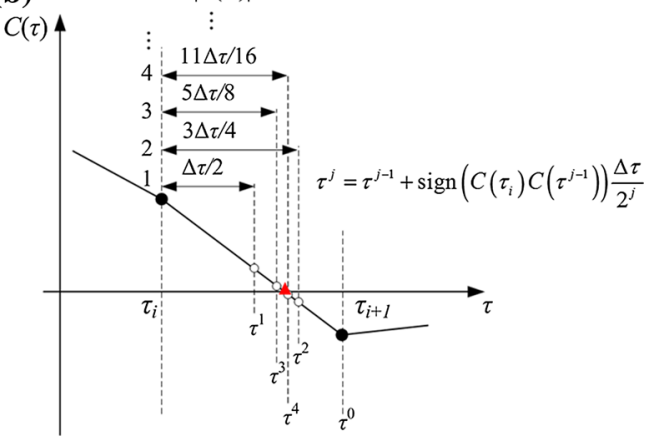

and variables

$\tau=\Omega_{0} t, \quad x_{1}=\frac{k}{P_{f}} X_{1}, \quad x_{2}=\frac{k}{P_{f}} X_{2}$,

$v_{1}=\frac{\mathrm{d} x_{1}}{\mathrm{~d} \tau}=\frac{k}{\Omega_{0} P_{f}} \dot{X}_{1}$,

$v_{2}=\frac{\mathrm{d} x_{2}}{\mathrm{~d} \tau}=\frac{k}{\Omega_{0} P_{f}} \dot{X}_{2}$.

Then the equations of motion are rewritten as

$$
\begin{aligned}
\dot{x}_{1}(\tau)= & v_{1}(\tau), \\
\dot{v}_{1}(\tau)= & \alpha \cos (\omega \tau)+f_{i}, \\
\dot{x}_{2}(\tau)= & v_{2}(\tau), \\
\dot{v}_{2}(\tau)= & \frac{1}{\gamma}\left(f_{i}+\left(1-\delta_{v}\right) s_{v}+\delta_{v} h_{f} s_{f}\right. \\
& \left.+\delta_{v}\left(1-h_{f}\right) f_{i}\right),
\end{aligned}
$$

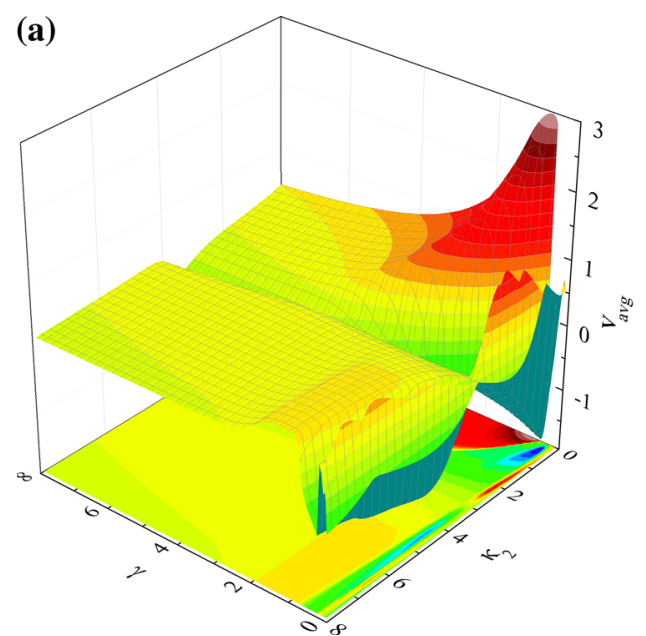

Fig. 3 Average progression velocity of the capsule $v_{\text {avg }}$ under varying mass ratio $\gamma$ and stiffness ratio $\kappa_{2}$ calculated for $\omega=$ $1.1, \alpha=1.6, \zeta=0.05, \delta_{1}=0.02, \delta_{2}=0.02$ and $\kappa_{1}=3$. The results of calculations are plotted using three-dimensional sur- where

$$
\begin{aligned}
f_{i}= & -2 \zeta\left(v_{1}(\tau)-v_{2}(\tau)\right)-\left(x_{1}(\tau)-x_{2}(\tau)\right) \\
& -h_{1} \kappa_{1}\left(x_{1}(\tau)-x_{2}(\tau)-\delta_{1}\right)-h_{2} \kappa_{2}\left(x_{1}(\tau)\right. \\
& \left.-x_{2}(\tau)+\delta_{2}\right), \\
h_{1}= & H\left(x_{1}(\tau)-x_{2}(\tau)-\delta_{1}\right), \\
h_{2}= & H\left(x_{2}(\tau)-x_{1}(\tau)-\delta_{2}\right), \\
h_{f}= & H\left(\left|f_{i}\right|-1\right), \\
\delta_{v}= & \delta\left(v_{2}(\tau)\right), \\
s_{v}= & \operatorname{sign}\left(v_{2}(\tau)\right), \\
s_{f}= & \operatorname{sign}\left(f_{i}\right) .
\end{aligned}
$$

It is worth noting that the non-smooth functions, such as the Heaviside, the Dirac Delta, and the sign functions, have significant influence on simulations. In

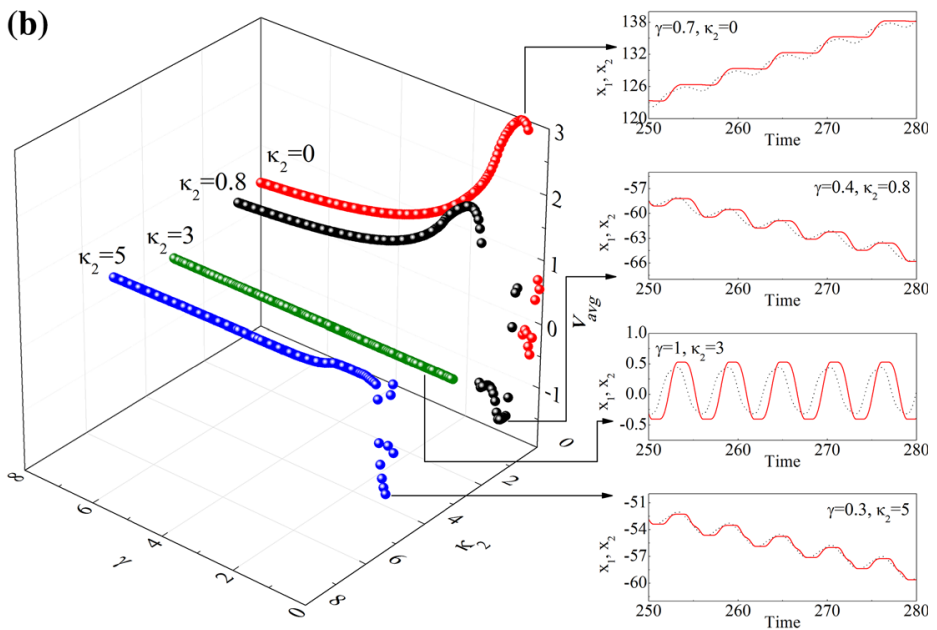

face in (a) with specific values of spring stiffness $\kappa_{2}$ presented in (b). Additional windows demonstrate the time histories of displacements of the internal mass (black dash line) and the capsule (red solid line). (Color figure online) 
order to obtain accurate results for numerical simulations, it is important to locate the critical non-smooth points precisely. Here, we adopted the bisection method [30] in the Runge-Kutta simulation, which automatically varies time step to locate the non-smooth points. A schematic illustration of the method is displayed in Fig. 2, where $C(\tau)$ represents the variable of the sign function and $C\left(\tau_{c}\right)=0$ is the switching points (e.g. $C(\tau)=X_{1}-G_{1}-X_{2}=0$ represents the inner mass just impacts the right plate). Figure $2 \mathrm{a}$ shows the traditional Runge-Kutta method with constant time step $\Delta \tau$, which skips the critical time instant $\tau_{c}$ between $\tau_{i}$ and $\tau_{i+1}$. In order to accurately locate $\tau_{c}$, the bisection algorithm was implemented to adjust the time step once the program detected $C\left(\tau_{i}\right) C\left(\tau_{i+1}\right)<0$. With $\tau^{0}=\tau_{i+1}, \tau^{j}$ is updated using

$\tau^{j}=\tau^{j-1}+\operatorname{sign}\left(C\left(\tau_{i}\right) C\left(\tau^{j-1}\right)\right) \frac{\Delta \tau}{2^{j}}$

until $C\left(\tau^{j}\right)$ is sufficiently close to zero, i.e. $\left|C\left(\tau^{n}\right)\right|<$ $\epsilon$, where $\epsilon$ is a small positive number given before the simulation. Finally, one can obtain $\tau_{c}=\tau^{n}$, which is the critical non-smooth point.

\section{Bifurcation analysis}

In order to gain an understanding of the system dynamics and optimise the progression speed of the capsule, bifurcation analysis is carried out next using the bifurcation diagram where the relative velocity $v_{1}^{*}-v_{2}^{*}$, which is a projection of the Poincare map on the $v_{1}-v_{2}$ axis, is plotted as a function of the control parameters, including mass ratio, stiffness ratios, gaps of contact, and frequency and amplitude of excitation. The calculations were run for 300 cycles of external excitation, and the data for the first 280 cycles were omitted to ensure steady state response, where the next 20 values of the velocity $v_{1}^{*}-v_{2}^{*}$ were plotted. The average progression of the capsule

$v_{\mathrm{avg}}=\frac{1}{N T}\left[x_{2}(N T)-x_{2}(0)\right]$,

where $N$ is the number of cycles and $T=\frac{2 \pi}{\omega}$ is one period of external excitation, was monitored for the purpose of optimisation. Here, the sign of $v_{\text {avg }}$ indicates whether the capsule moves forward (positive $v_{\text {avg }}$ ) or backward (negative $v_{\text {avg }}$ ). In addition, abbreviations are used to describe periodic motion of the system, e.g. P-12-3 represents a period-1 motion with two left impacts and three right impacts per period of external excitation.

\subsection{Influence of mass ratio}

To investigate the influence of the mass ratio $\gamma$ and the spring stiffness $\kappa_{2}$ on the average progression of
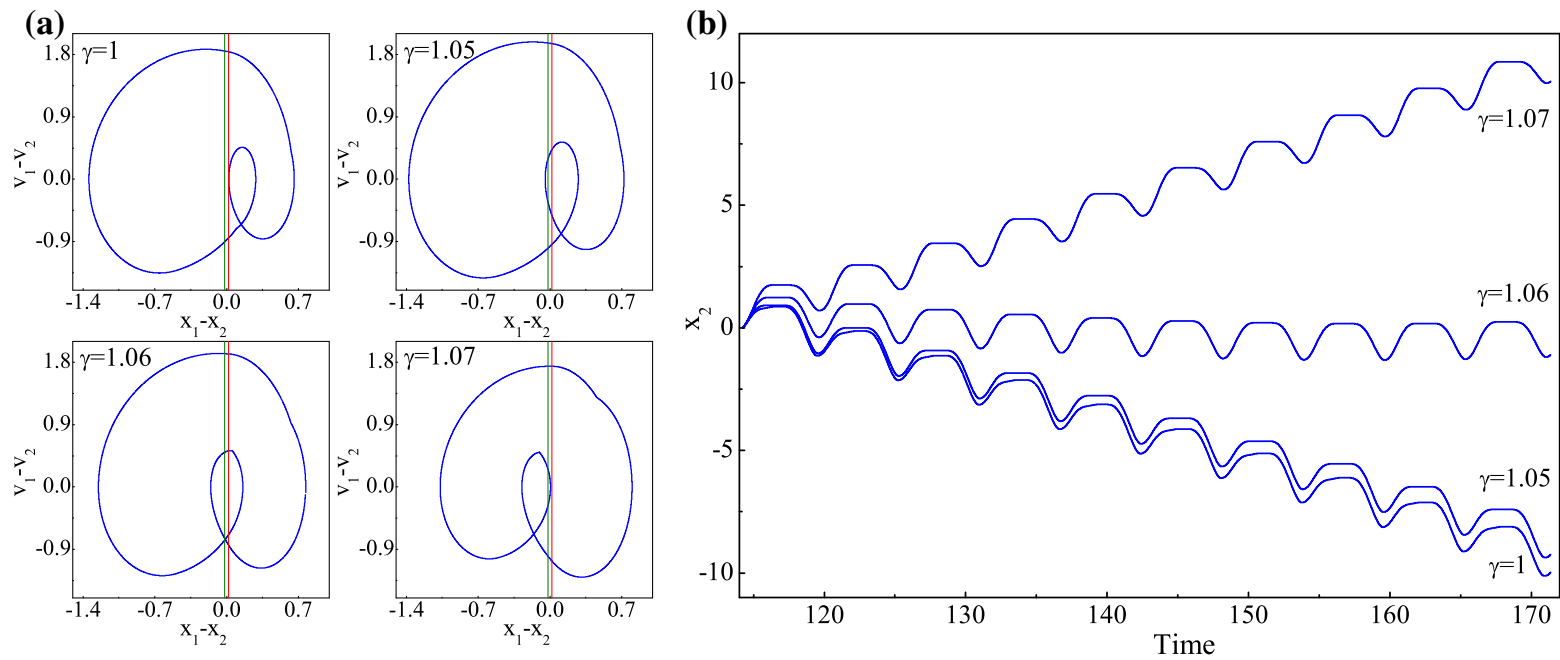

Fig. 4 a Trajectories on the phase plane $\left(x_{1}-x_{2}, v_{1}-v_{2}\right)$ and b time histories of displacements of the capsule calculated for $\kappa_{2}=0.8$. The locations of the left and right impact surfaces are shown by green and red lines, respectively. (Color figure online) 


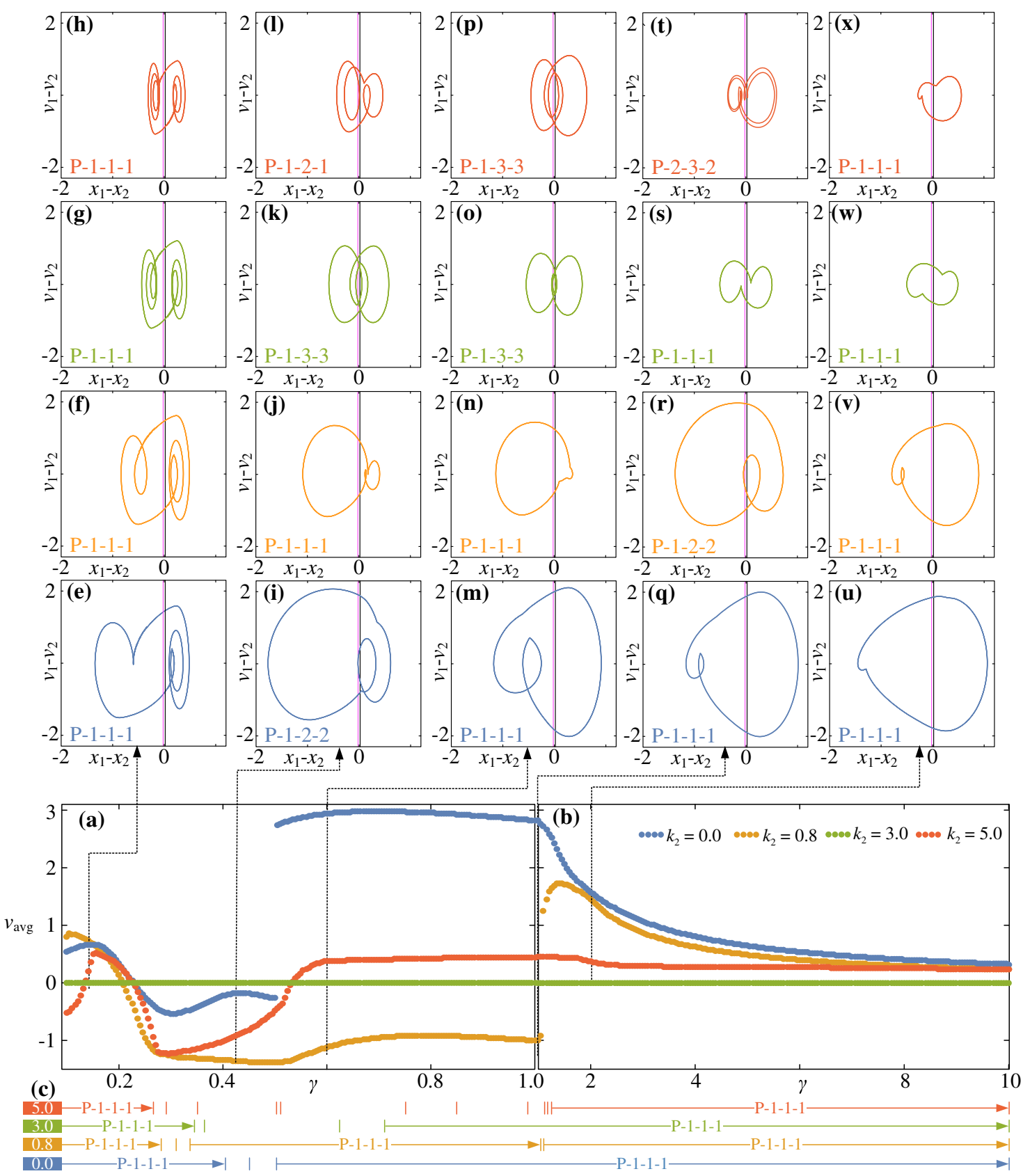

(d) $\gamma_{1} \longrightarrow \mathrm{P}-m-n-l \rightarrow \gamma_{2}:$ Periodic- $m$ motion with $n$ left impacts and $l$ right impacts are observed for $\gamma \in\left[\gamma_{1}, \gamma_{2}\right]$

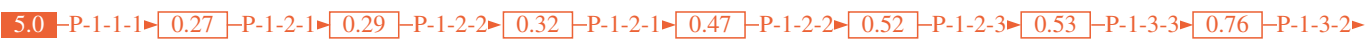

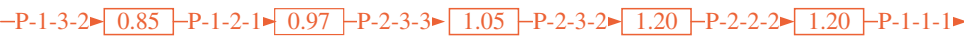

$3.0-\mathrm{P}-1-1-1 \curvearrowleft 0.35-\mathrm{P}-1-2-2 \backsim 0.37-\mathrm{P}-1-3-3 \backsim 0.62-\mathrm{P}-1-2-2 \backsim 0.72-\mathrm{P}-1-1-1 \sim$

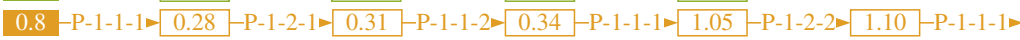

$0.0-\mathrm{P}-1-1-1 \backsim 0.41-\mathrm{P}-1-1-2-0.45-\mathrm{P}-1-2-2-0.51-\mathrm{P}-1-1-1$

Fig. 5 Average progression velocity of the capsule as a function of mass ratio $\mathbf{a} \gamma \in[0.1,1.0]$ and $\mathbf{b} \gamma \in[1.0,10]$ calculated for $\omega=1.1, \alpha=1.6, \zeta=0.05, \delta_{1}=0.02, \delta_{2}=0.02, \kappa_{1}=3$ with $\kappa_{2}=0.0$ (blue dots), 0.8 (orange dots), 3.0 (green dots) and 5.0 (red dots). Critical bifurcation points for the capsule sys- tem are marked and recorded in (c) and (d). Additional windows e-u demonstrate the trajectories of the capsule system on the phase plane. Locations of the left and the right impact surfaces are shown by purple and black lines, respectively. (Color figure online) 
the capsule, numerical simulations were carried out for $\gamma \in[0,8]$ and $\kappa_{2} \in[0,8]$, and the calculated results are presented in Fig. 3. It can be seen from Fig. $3 \mathrm{a}$ that, the maximum velocity is achieved by the capsule with one-sided constraint $\left(\kappa_{2}=0\right)$, and the average velocity of the capsule decreases as the spring stiffness $\kappa_{2}$ increases. In Fig. 3b, specific values of spring stiffness $\kappa_{2}$ are shown, where the calculations for $\kappa_{2}=0,0.8,3,5$ are denoted by red, black, green, and blue, respectively. It can be observed that, except the symmetrical condition $\left(\kappa_{1}=\kappa_{2}=3\right)$, there is an immediate directional change of capsule progression from backward to forward as mass ratio increases, which is due to the grazing when the inner mass contacts with the two-sided constraints. Figure 4 demonstrates the occurrence of such grazing when $\kappa_{2}=0.8$. It can be seen from Fig. 4a that, the system bifurcates from P-1-1-1 $(\gamma=1)$ to P-1-2-2 $(\gamma=1.05)$, and then from P-1-2-2 $(\gamma=1.06)$ to P-1-2-1 $(\gamma=1.07)$. Figure $4 \mathrm{~b}$ presents the displacements of the capsule from backward to forward progression owing to this grazinginduced bifurcation.

A further investigation of influence of the mass ratio $\gamma$ and the spring stiffness $\kappa_{2}$ on the average velocity of the capsule was carried out, and the calculated results are shown in Fig. 5. As can be seen from these figures, at $\gamma=0.1$, Fig. 5e-h correspond to $\kappa_{2}=0.0,0.8,3.0$

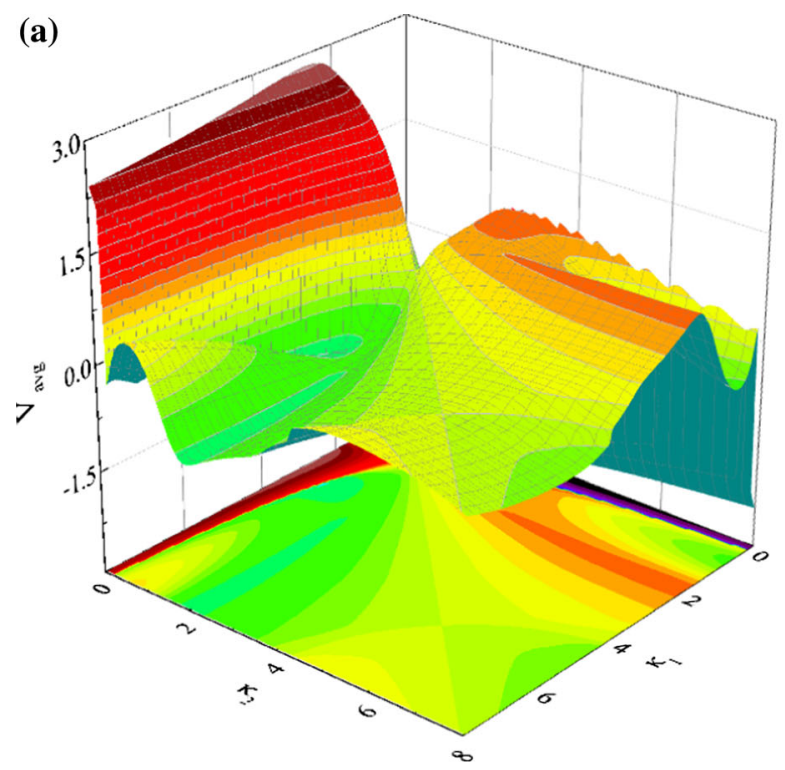

and 5.0, respectively. The corresponding motions are all P-1-1-1 and the capsule moves forward. Increasing the mass ratio to 0.45 , one obtains negative velocities with the capsule motions becoming P-1-2-2, P-1-1-1, P-1-3-3, and P-1-2-1 as illustrated in Fig. 5i-k and (1), respectively. Here, although they are all period-1 motions, the number of impacts per period of external excitation increases. For example, for $\kappa_{2}=3$, the capsule system bifurcates from P-1-1-1 to P-1-3-3 as the mass ratio increases from 0.1 to 0.45 . It can be observed from Fig. 5a that, as the mass ratio further increases, the average velocity of the capsule increases drastically and achieves its maximum for the capsule with one-sided constraint $\left(\kappa_{2}=0.0\right)$, while the average velocity of the capsule experiences a continuous rise to a small positive value when the left spring is stiffer $\left(\kappa_{2}=5.0\right)$. However, such phenomenon cannot be observed when the stiffness of the left spring is weak $\left(\kappa_{2}=0.8\right)$ or symmetrical $\left(\kappa_{2}=3.0\right)$. When $\kappa_{2}=0.8$, the drastic change of the average velocity from negative to positive is recorded at $\gamma \approx 1.06$. By comparing Fig. 5i with (m) and (r) with (v), one may notice that both drastic changes are due to grazing bifurcations as illustrated in Fig. 4. From Fig. 5t, we can observe a period-2 motion (P-2-3-2) which indicates that stiffer spring may induce period doubling of the capsule system. As the mass ratio increases, the inner mass has less

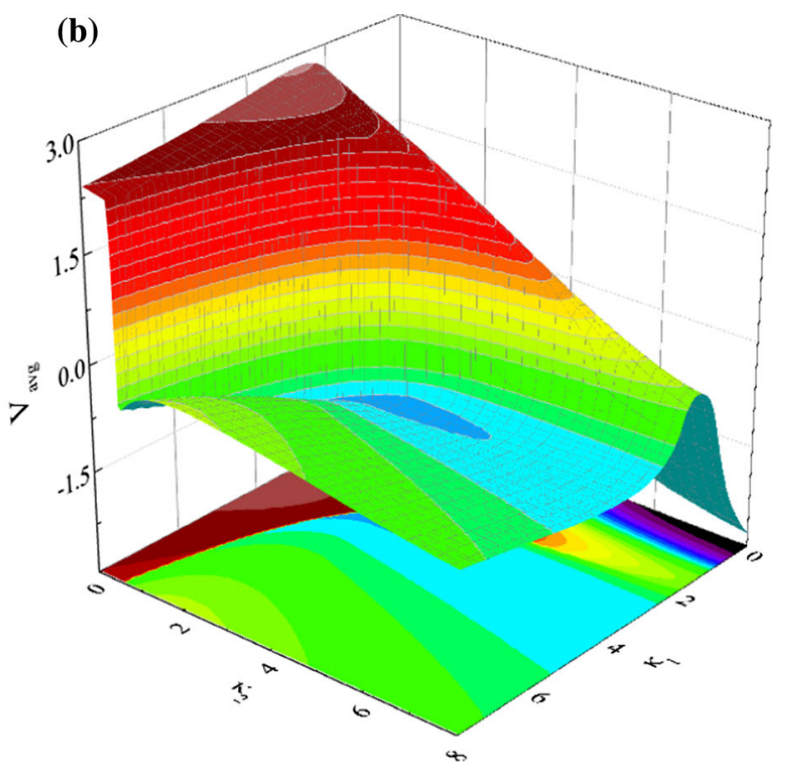

Fig. 6 Average progression velocity of the capsule $v_{\text {avg }}$ under varying the stiffness ratios, $\kappa_{1}$ and $\kappa_{2}$ calculated for $\omega=1.1, \alpha=$ $1.6, \zeta=0.05, \delta_{1}=0.02, \gamma=1, \mathbf{a} \delta_{2}=0.02$, and $\mathbf{b} \delta_{2}=1$ 

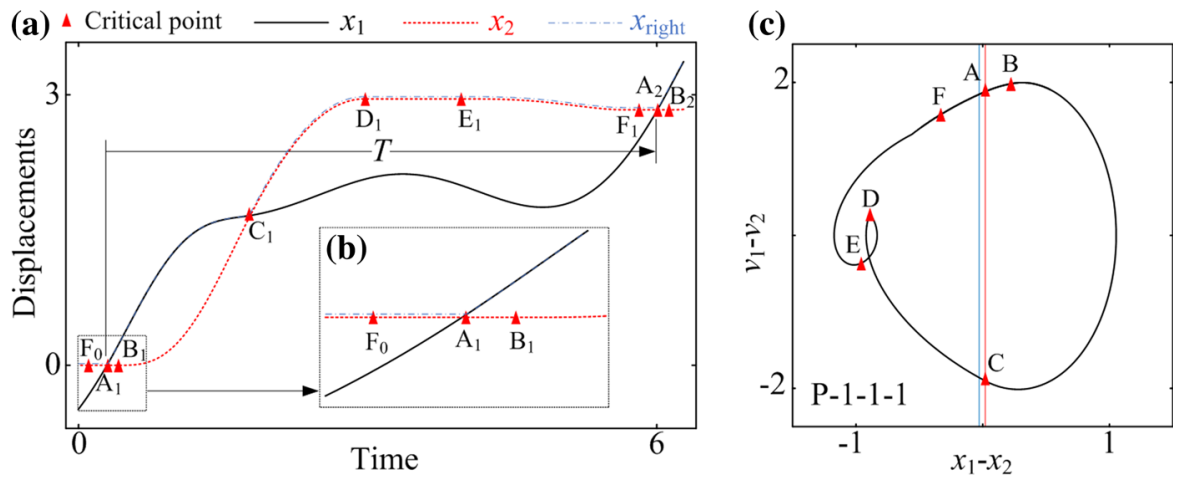

Fig. 7 a Time histories of the inner mass $x_{1}$ (black solid line), the capsule $x_{2}$ (red dash line), and the right plate $x_{\text {right }}$ (blue dashdot line), $\mathbf{b}$ a blow-up window showing the transition from the phase of stationary capsule without contact of the right plate to the phase of stationary capsule with contact, and $\mathbf{c}$ the trajectory on the phase plane $\left(x_{1}-x_{2}, v_{1}-v_{2}\right)$, corresponding to the maximum progression speed obtained for $\omega=1.1, \alpha=1.6, \zeta=$ $0.05, \delta_{1}=0.02, \delta_{2}=0.02, \gamma=1, \kappa_{1}=2.7$, and $\kappa_{2}=0.0$. Locations of the left and the right impact surfaces are shown by blue and red lines, respectively. (Color figure online)
Fig. 8 Time histories and phase trajectories of the capsule system computed for $\omega=1.1, \alpha=1.6, \zeta=$ $0.05, \delta_{1}=0.02, \delta_{2}=$ $1, \gamma=1, \kappa_{1}=2.7,(\mathbf{a}, \mathbf{b})$ $\kappa_{2}=0,(\mathbf{c}, \mathbf{d}) \kappa_{2}=0.8$, and $(\mathbf{e}, \mathbf{f}) \kappa_{2}=5.0$. Locations of the left and the right impact surfaces are shown by blue and red lines, respectively. (Color figure online)
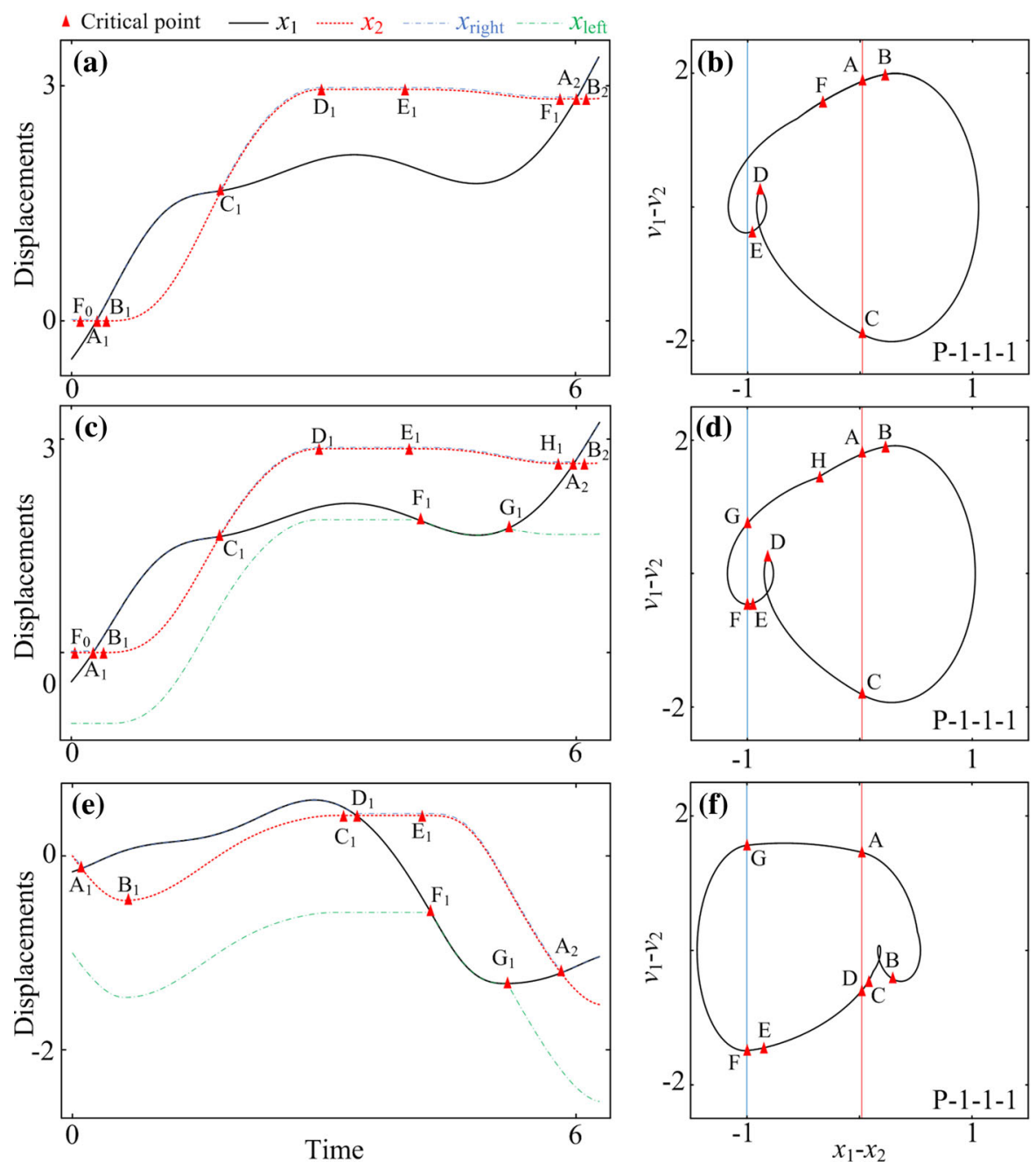
effect on the dynamics of the capsule, so all the velocities gradually decline to zero regardless of the values of $\kappa_{2}$. In addition, it is worth noting that, by comparing all the trajectories on the phase plane in Fig. 5, the left spring can effectively constrain the stroke length of the inner mass when its stiffness is sufficiently large.

From a control point of view, it is more effective to control the direction of capsule progression when the weight of the capsule is less than or equal to the weight of the internal mass $(\gamma \leq 1)$ providing that the two-sided constraints are asymmetrical $\left(\kappa_{1} \neq \kappa_{2}\right)$. The control strategy for the capsule system could be to alter its mass ratio slightly around $\gamma=1$ for controlling its progression direction by using a weak left constraint (e.g. $\kappa_{2}=0.8$ ), which offers both forward and backward progressions at acceptable average speeds.
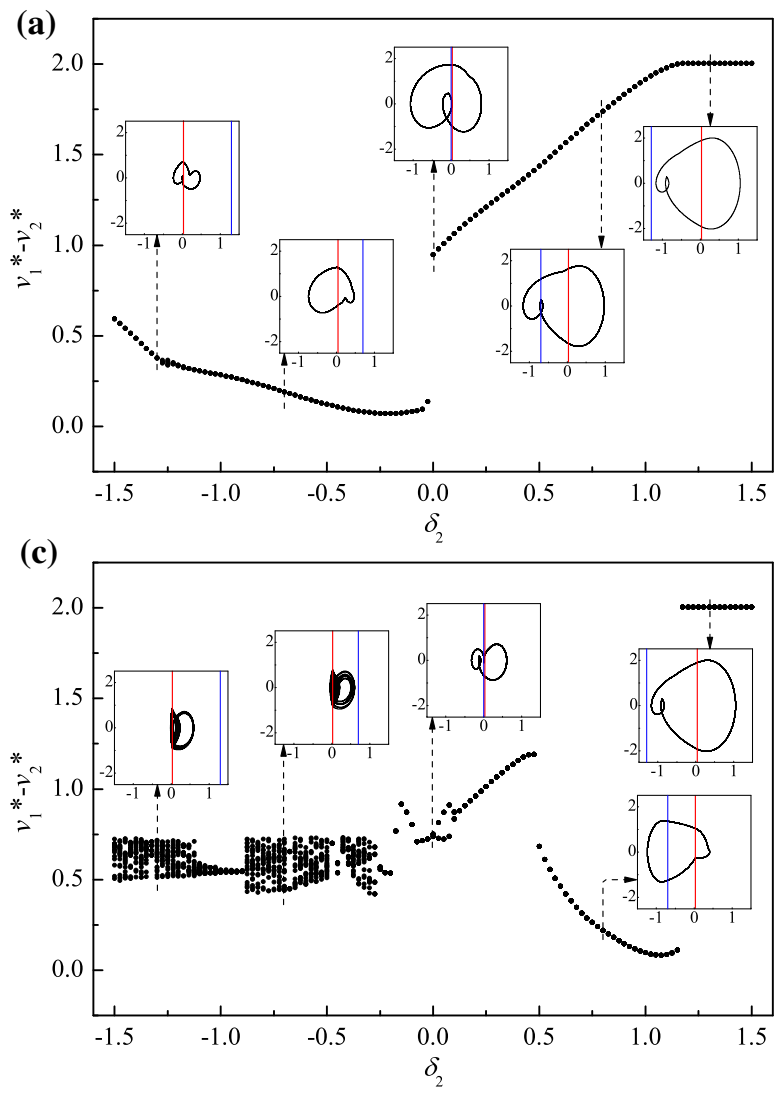

Fig. 9 a Bifurcation diagram and $\mathbf{b}$ average progression velocities under variation of gap $\delta_{2}$ calculated for $\omega=1.1, \alpha=$ $1.6, \zeta=0.05, \kappa_{1}=2.7, \kappa_{2}=0.8, \delta_{1}=0.02, \gamma=1$. c Bifurcation diagram and $\mathbf{d}$ average progression velocities under variation of gap $\delta_{2}$ calculated for $\omega=1.1, \alpha=1.6, \zeta=0.05, \kappa_{1}=$ $2.7, \kappa_{2}=5.0, \delta_{1}=0.02, \gamma=1$. Additional windows demon-

\subsection{Influence of stiffness ratios}

Average progression velocity of the capsule under varying the stiffness ratios, $\kappa_{1}$ and $\kappa_{2}$ are presented in Fig. 6a, where the gaps of contact for both springs are equal $\left(\delta_{1}=\delta_{2}=0.02\right)$. As can be seen from this figure, the best progression is achieved by the capsule system with one-sided constraint $\left(\kappa_{2}=0\right)$, and the maximal average velocity is recoded at $\kappa_{1}=2.7$ and $\kappa_{2}=0$. Figure 7a shows the time histories of displacements of the inner mass and the capsule, and Fig. 7c presents the capsule trajectory on the phase plane $\left(x_{1}-x_{2}, v_{1}-v_{2}\right)$ for the maximal average velocity recorded in Fig. 6a. A blow-up window in Fig. 7b clearly shows the displacements of the mass, the capsule, and the right plate transiting from the phase of stationary capsule without
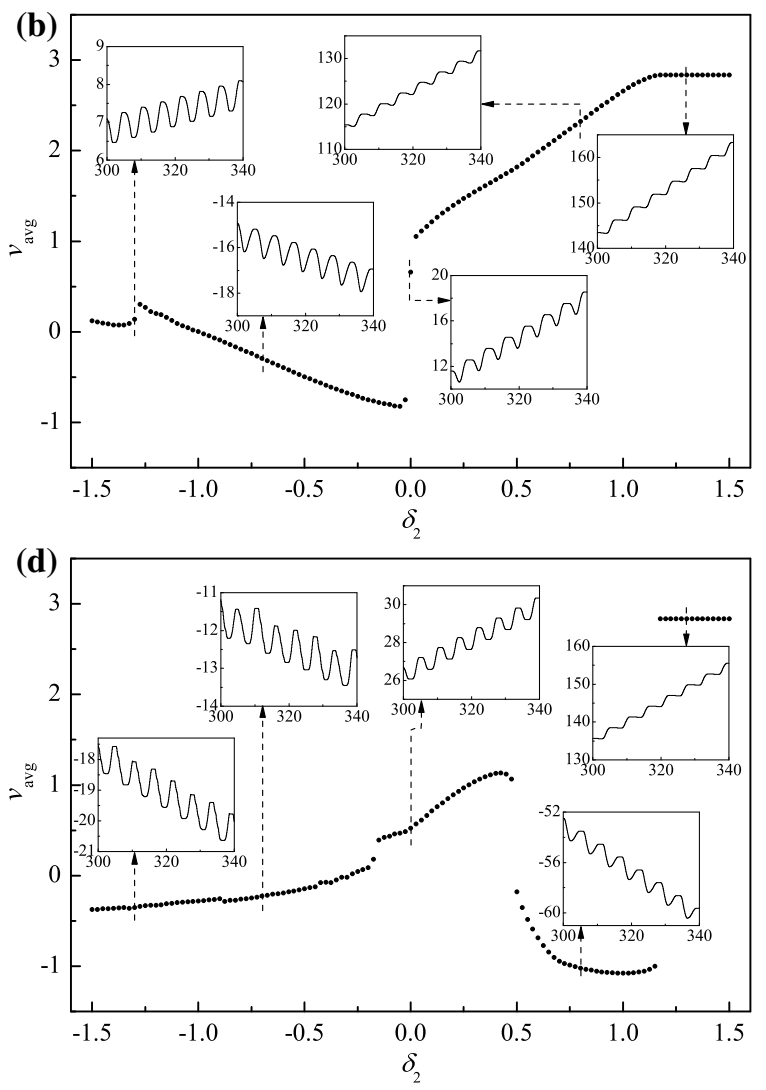

strate the trajectories on the phase plane $\left(x_{1}-x_{2}, v_{1}-v_{2}\right)$ and times histories of capsule displacements obtained for $\delta_{2}=$ $-1.3, \delta_{2}=-0.7, \delta_{2}=0, \delta_{2}=0.8$, and $\delta_{2}=1.3$. Locations of the impact surfaces for the right and the left constraints are shown by red and blue lines, respectively. (Color figure online) 
contact of the right plate to the one with contact. It can be seen that the maximum speed is achieved by P-1$1-1$, and an entire time period $(T)$ of the motion has been divided into six phases. The first phase begins at Point A, where the inner mass just contacts with the right plate, but the elastic forces on the capsule are too small to overcome external frictional resistance until Point B is reached. Thereafter, the capsule accelerates to progress before Point $\mathrm{C}$, where the mass is separated from the right plate and the capsule is decelerated by the friction. The velocity of the capsule reduces to zero at Point $\mathrm{D}$ and the capsule is stationary until Point $\mathrm{E}$. At Point E, the elastic forces on the capsule exceed the threshold of the static friction, and a slight backward motion of the capsule is observed. At Point F, the velocity of the capsule becomes zero again, and the capsule has a short period of sticking phase until Point A.

The capsule progression for unsymmetrical gaps of contact ( $\delta_{1}=0.02$ and $\left.\delta_{2}=1\right)$ was studied, and the calculated result is presented in Fig. 6b. It can be seen from the figure that one-sided constraint (i.e. $\kappa_{2}=0$ or $\left.\kappa_{1}=0\right)$ is the best choice for both forward and backward progressions. A detailed behaviour of the capsule system are shown in Fig. 8, where the capsules with one-sided constraint $\left(\kappa_{2}=0\right)$, two-sided constraints with weak left spring $\left(\kappa_{2}=0.8\right)$, and two-sided constraints with strong left spring $\left(\kappa_{2}=5\right)$ are plotted in Fig. 8. Comparing the cases for one-sided and twosided constraints with weak left spring, the trajectories in Fig. 8a-d are similar until Point $\mathrm{F}$ at where the inner mass contacts with the left plate and the capsule has a slightly larger backward displacement thereafter. Therefore, the velocity of the capsule can be lowered by inducing the left spring. Moreover, when the stiffness of the left spring becomes greater $\left(\kappa_{2}=5.0\right)$, large backward progression of the capsule encounters, and the overall progression of the capsule becomes negative as shown in Fig. 8e, $\mathrm{f}$.

An investigation on the gap of left constraint was carried out, and the comparison between the weak left constraint $\left(\kappa_{2}=0.8\right)$ and the strong one $\left(\kappa_{2}=5.0\right)$ is shown in Fig. 9. Here, we calculated the gap of the
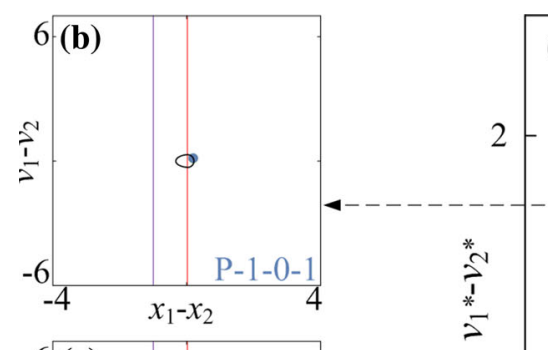

(a)
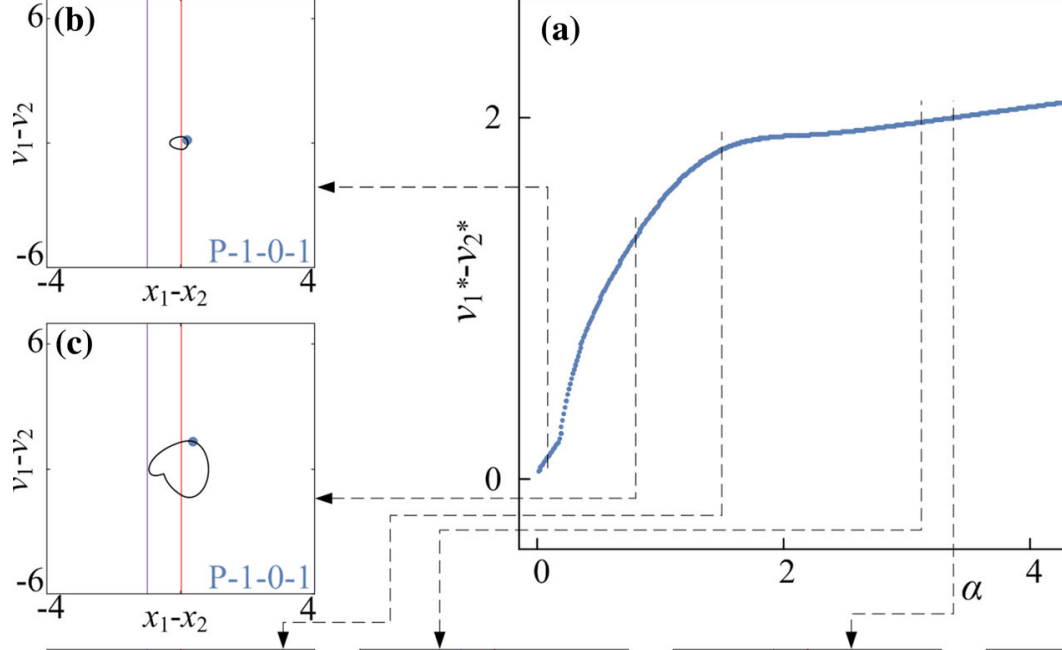
left constraint for $\delta_{2} \in[-1.5,1.5]$, where the negative value of the gap indicates a prestressed constraint. As can be seen from the figure, when the gap decreases from $\delta_{2}=1.5$, the internal mass begins to impact left constraint at $\delta_{2}=1.175$. As shown in Fig. 9b, d, the grazing of such impact alters the direction of capsule progression from forward to backward for the capsule with strong left constraint, while it only affects the forward speed of the capsule with weak left constraint. As the gap decreases, the capsule with weak left constraint encounters this sudden change of progression direction owing to the grazing contact with the left con- straint at $\delta_{2} \approx 0$. On the other hand, the capsule with strong left constraint experiences a period and a reverse period doubling at $\delta_{2}=0.125$ and -0.125 , respectively. When the left constraint is prestressed $\left(\delta_{2}<0\right)$, comparing Fig. 9a, c, the capsule with weak constraint has period-1 motion, while the one with strong constraint experiences chaotic motion. It can be found from Fig. $9 \mathrm{~b}$,d, both average progressions are slow. However, as presented in the additional windows of phase trajectories, the stroke length of the internal mass under prestressed condition is significantly reduced comparing to the one with right constraint only.

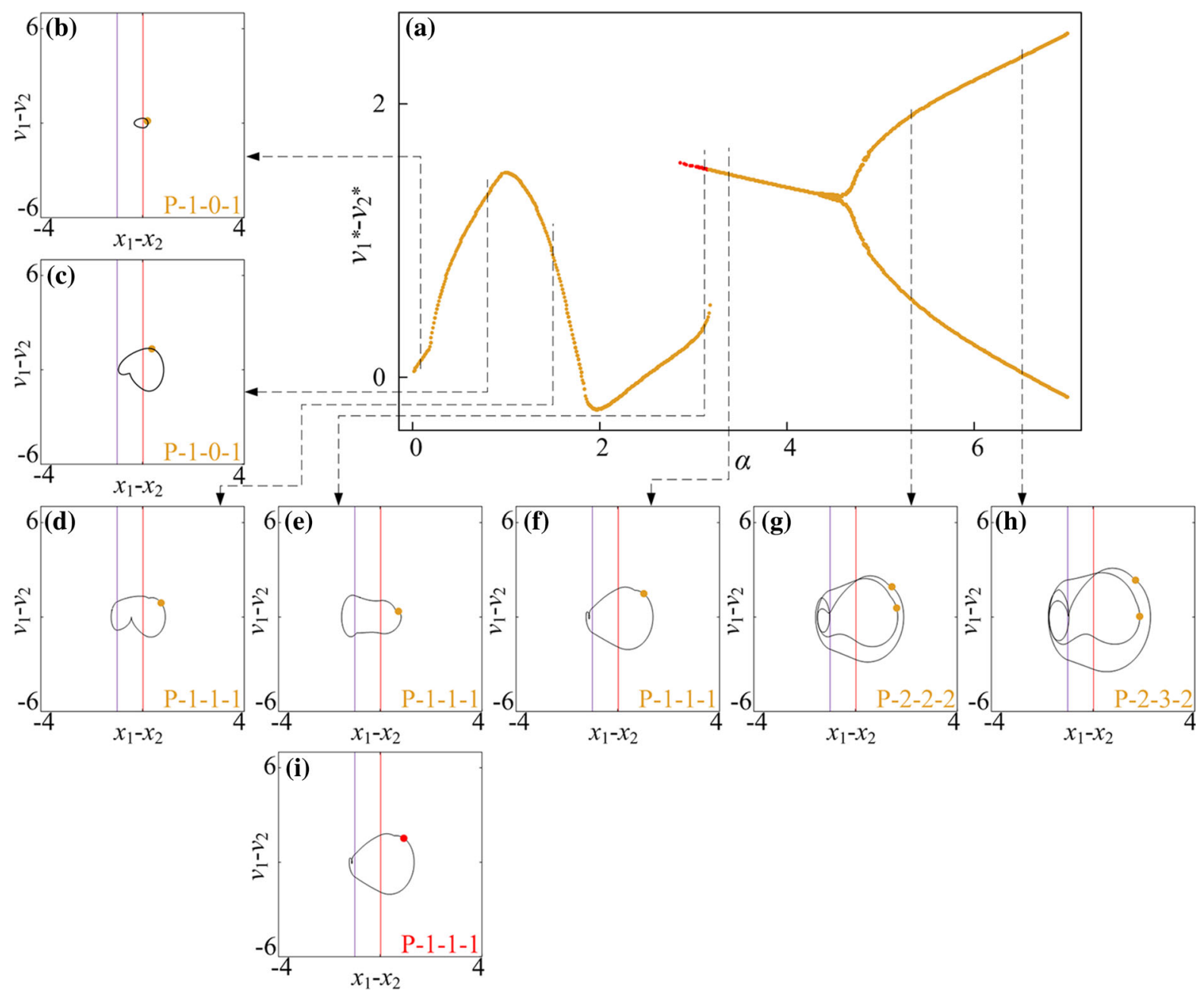

Fig. 11 a Bifurcation diagram under variation of amplitude of excitation $\alpha$ calculated for $\omega=1.1, \zeta=0.05, \gamma=1, \delta_{1}=$ $0.02, \delta_{2}=1.0, \kappa_{1}=2, \kappa_{2}=5$. b-i Trajectories of the system displayed on the phase plane $\left(x_{1}-x_{2}, v_{1}-v_{2}\right)$ for $\alpha=$ $0.10,0.80,1.50,3.12,3.37,5.43$ and 6.50 . Coexisting attractors are marked by red dots. Locations of the left and the right impact surfaces are shown by purple and red lines, respectively. Poincaré sections are marked by orange and red dots on the phase plane. (Color figure online) 


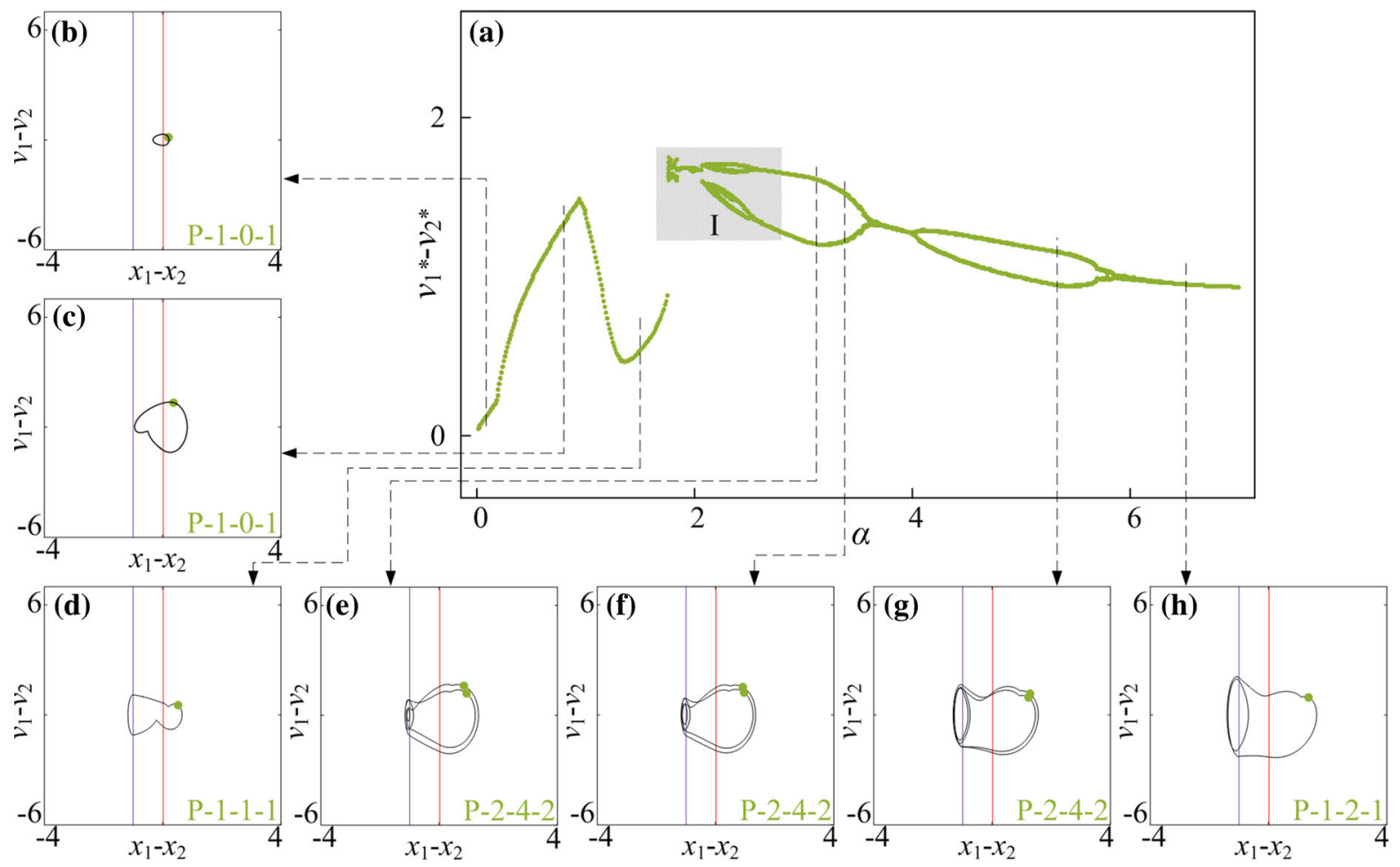

Fig. 12 a Bifurcation diagram under variation of amplitude of excitation $\alpha$ calculated for $\omega=1.1, \zeta=0.05, \gamma=1, \delta_{1}=$ $0.02, \delta_{2}=1.0, \kappa_{1}=2, \kappa_{2}=20 . \mathbf{b}-\mathbf{h}$ Trajectories of the system displayed on the phase plane $\left(x_{1}-x_{2}, v_{1}-v_{2}\right)$ for

Based on the discussions above, it can be drawn that the maximum positive progression velocity can be achieved by the capsule with one-sided right constraint, and the introduction of the left spring with any value of stiffness may reduce its progression speed. Nevertheless, if the miniaturization of the capsule is required, the prestressed weak constraint could be a viable option.

\subsection{Influence of amplitude of excitation}

Bifurcation diagrams of the capsule system with $\kappa_{2}=$ $0,5,20$ under variation of amplitude of excitation $\alpha$ are presented in Figs. 10, 11, and 12, respectively. Additional windows in these figures demonstrate the capsule trajectories on the phase plane $\left(x_{1}-x_{2}, v_{1}-v_{2}\right)$. One can observe that the bifurcation diagram shown in Fig. 10 for the capsule with one-sided constraint $\left(\kappa_{2}=0\right)$ is smooth, and all its corresponding phase trajectories are P-1-1-1. For $\kappa_{2}=5$ shown in Fig. 11, the bifurcation curve is smooth until $\alpha=2.86$ at where an immedi- $\alpha=0.10,0.80,1.50,3.12,3.37,5.43$ and 6.50. Locations of the left and the right impact surfaces are shown by purple and red lines, respectively. Poincaré sections are marked by green dots. (Color figure online)

ate increase in the relative velocity $v_{1}-v_{2}$ due to the grazing contact with the left spring is recorded, and two P-1-1-1 presented in Fig. 11e, i coexist for $\alpha \in$ [2.86, 3.18]. As the amplitude of excitation increases, a period doubling is observed at $\alpha=4.35$ and the capsule bifurcates from P-1-1-1 to P-2-2-2. When the left spring is much stiffer $\left(\kappa_{2}=20\right)$, the dynamic responses of the system become more complicated. The grazing event of the left plate is recorded at $\alpha=1.76$ followed by a period doubling cascade, which is shown in Fig. 12. As the amplitude of excitation increases, a reverse period doubling from P-2-4-2 to P-1-2-1 is observed at $\alpha=3.69$. Thereafter, a period and a reverse period doubling between P-2-4-2 and P-1-2-1 are recorded at $\alpha=4$ and 5.87, respectively.

In order to obtain a better insight into the dynamic responses of the stiffer left spring $\left(\kappa_{2}=20\right)$, Fig. 13 presents the bifurcations in the range marked by the number "I" in Fig. 12. As can be seen from this figure, the system starts with a quasi-periodic motion which is followed by a period- 3 and period- 6 motions. As 

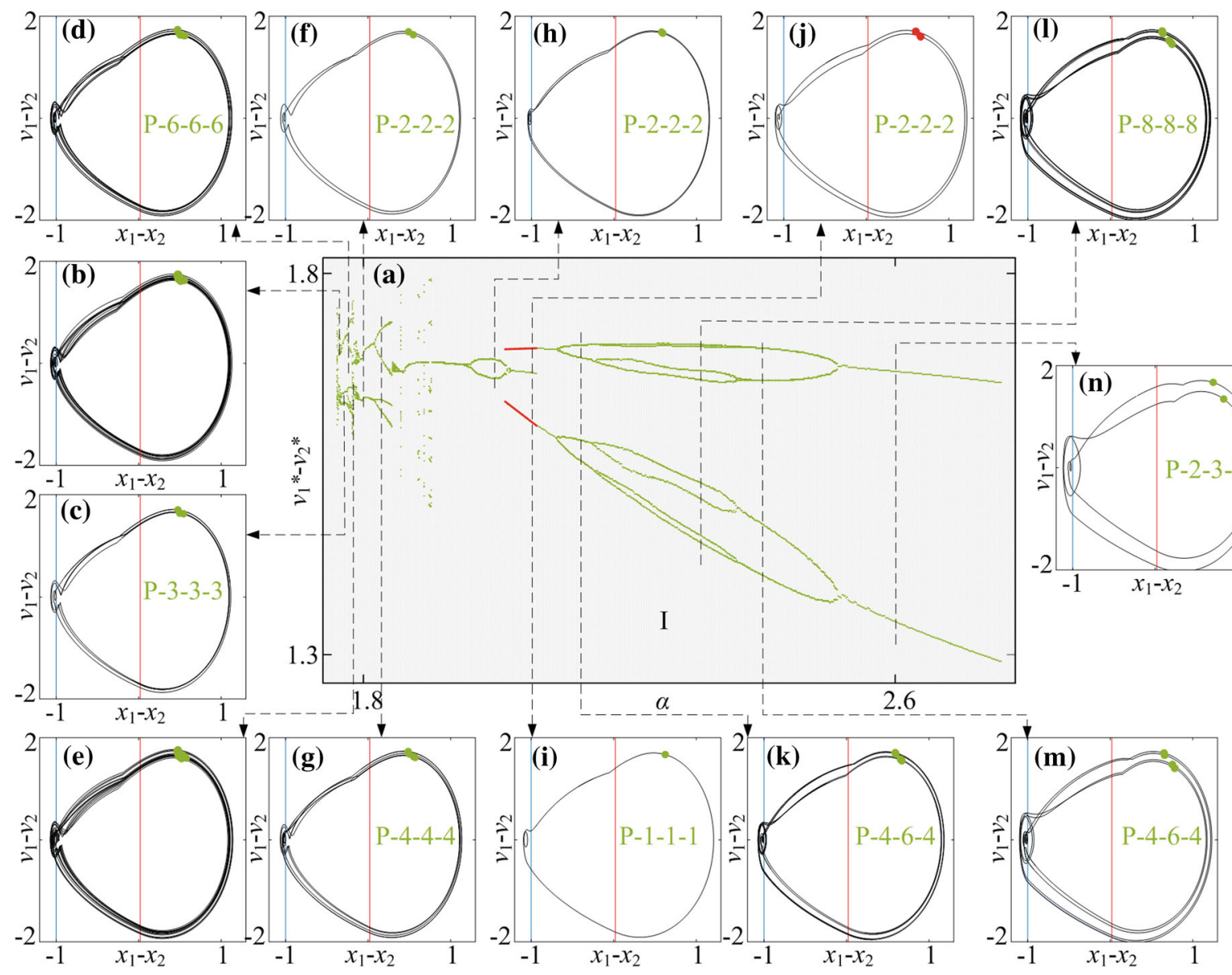

Fig. 13 a Bifurcation diagram in the range marked by the number "I" in Fig. 12, where coexisting attractors are marked by red dots. b-n Additional windows demonstrate the trajectories on the phase plane $\left(x_{1}-x_{2}, v_{1}-v_{2}\right)$ obtained for $\alpha=$ $1.763,1.768,1.780,1.785,1.8,1.820,2.0,2.056,2.056$ (coex- isting), 2.123, 2.312, 2.410, and 2.6. Locations of the left and the right impact surfaces are shown by blue and red lines, respectively. Poincaré sections are marked by green and red dots on the phase plane. (Color figure online) the amplitude of excitation increases, another quasiperiodic motion followed by a period- 2 and period-4 motions is observed. For a short range of amplitude of excitation, the coexistence of a P-1-1-1 and a P-22-2 motion is recorded where coexisting attractors are marked by red dots. From this point onwards, the capsule bifurcates from P-2-2-2 to P-4-6-4 and to P-8-8-8 both via a period doubling at $\alpha=2.323$ and 2.362, respectively. Thereafter, the capsule experiences two reverse period doublings at $\alpha=2.323$ and 2.362, consequently, the dynamic response of the system bifurcates from P-8-8-8 to P-2-3-2, and then P-2-4-2 afterwards.

The calculated average velocities of the capsule with $\kappa_{2}=0,5,20$ under variation of amplitude of excita- tion $\alpha$ are presented in Figs. 14, 15 and 16, respectively, with additional windows demonstrating the time histories of displacements of the inner mass (black solid line) and the capsule (red dash line). It can be seen from these figures that, the average velocities vary with respect to the increase of excitation amplitude. When the excitation amplitude is small, the capsule is stationary owing to the static friction from the supporting surface. As the excitation amplitude increases, all the systems experience P-1-0-1 responses with the same progression speeds until the inner mass firstly contacts with the left constraint at $\alpha=0.93$. Due to the effect of the left constraint, the capsule systems with twosided constraints ( $\kappa_{2}=5$ and 20 ) have lower average speeds than the one with one-sided constraint. Compar- 


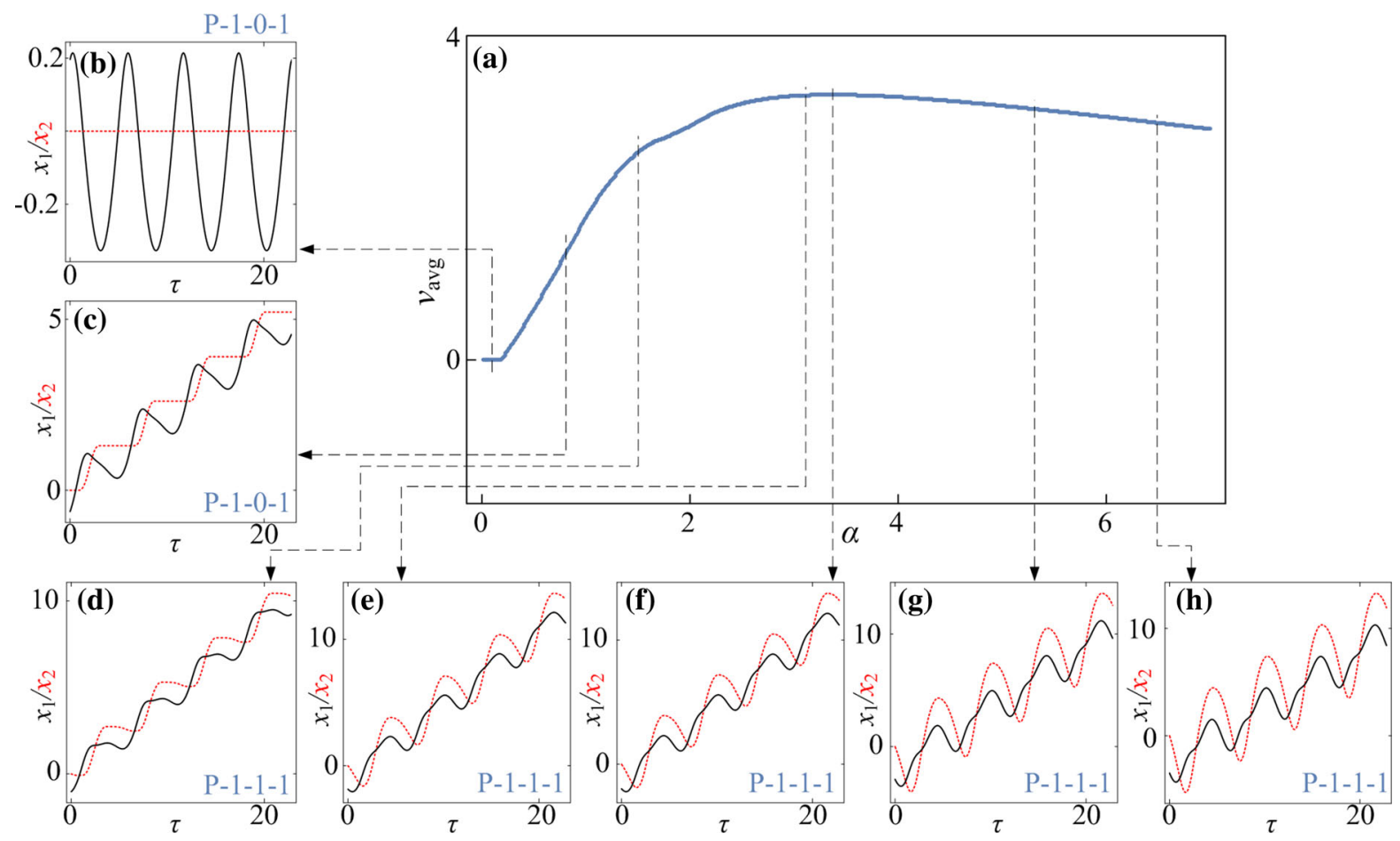

Fig. 14 a Average progression velocities under variation of amplitude of excitation $\alpha$ calculated for $\omega=1.1, \zeta=0.05, \gamma=$ $1, \delta_{1}=0.02, \delta_{2}=1.0, \kappa_{1}=2, \kappa_{2}=0$. b-h Additional windows demonstrate the time histories of displacements of the inner

ing their displacements in Figs. 14d, 15d and 16d, it can be noted that increase of stiffness of the left spring may cause fluctuation of the inner mass leading to backward motion of the capsule. It is worth noting that a forward P-1-1-1 coexists with a backward P-1-1-1 for a short range of amplitude of excitation for $\kappa_{2}=5$ due to the grazing contact with the left constraint. For one-sided constraint $\left(\kappa_{2}=0\right)$, the maximum velocity of the capsule is achieved by a P-1-1-1 motion at $\alpha=3.37$. For $\kappa_{2}=20$, the capsule with two-sided constraints reaches its maximum velocity at $\alpha=5.34$ by a P-2-4-2 motion, which is higher than the system with one-sided constraint. With a weak left spring $\left(\kappa_{2}=5\right)$, it can be seen from Fig. 15h that, the maximal average speed obtained by the capsule through a P-2-3-2 motion is higher than the maximal speeds recorded for the other two systems. Here, it can be concluded that the best performance of the capsule system could be achieved by using a weak left spring under a larger amplitude of excitation. mass (black solid line) and the capsule (red dash line) obtained for $\alpha=0.10,0.80,1.50,3.12,3.37,5.43$ and 6.50. (Color figure online)

Figure 17 shows an interesting grazing bifurcation observed for $\kappa_{2}=20$ when the phase trajectory of the system makes grazing contact simultaneously with two discontinuity boundaries, $I_{1}:=\left(x_{1}-x_{2}\right)+\delta_{2}=0$ and $I_{2}:=\left(x_{1}-x_{2}\right)+2 \zeta\left(v_{1}-v_{2}\right)+\kappa_{2}\left(x_{1}-x_{2}+\delta_{2}\right)+1=0$, which define the impact of the left constraint and the transition to backward drift, respectively. As can be seen from Fig. 17a, the capsule has forward drift only when its phase trajectory does not contact the discontinuity boundaries at $\alpha=0.91$. As the amplitude of excitation increases to $\alpha=0.95$, two grazing contacts occur and the backward drift of the capsule appears at every cycle. Another interesting bifurcation, namely the boundary-intersection crossing bifurcation [19], is observed for $\kappa_{2}=5$ which is presented in Fig. 18. One can observe from Fig. 18a that, when the capsule is stationary $I_{2}:=v_{2}=0$, the trajectory of the capsule on the phase plane $\left(x_{1}-x_{2}, v_{2}\right)$ crosses the discontinuity boundary, $I_{0}:=\left(x_{1}-x_{2}\right)-\delta_{1}=0$, which defines the impact of the right constraint. As shown in 


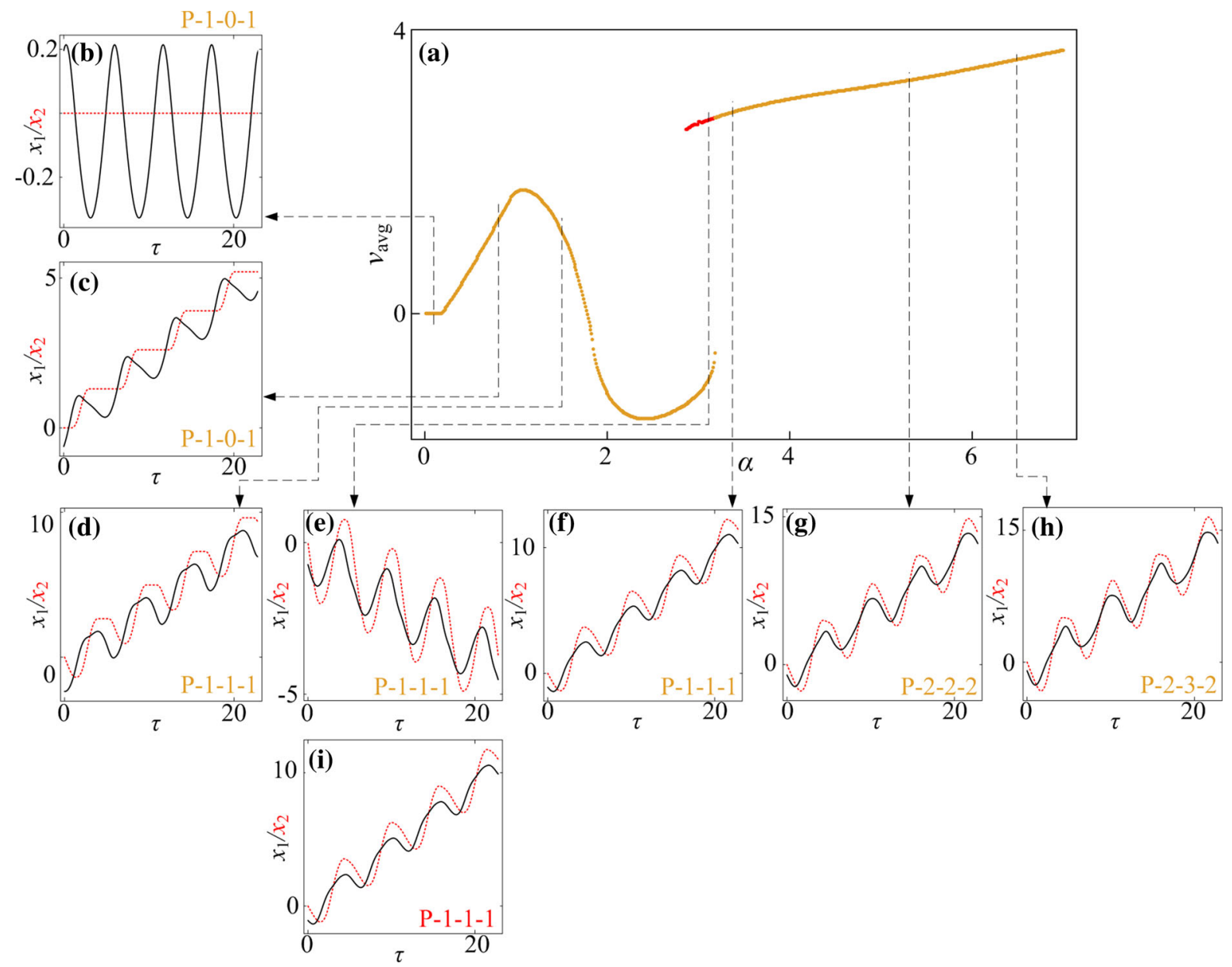

Fig. 15 a Average progression velocities under variation of amplitude of excitation $\alpha$ calculated for $\omega=1.1, \zeta=0.05, \gamma=$ $1, \delta_{1}=0.02, \delta_{2}=1.0, \kappa_{1}=2, \kappa_{2}=5$. b-i Additional windows demonstrate the time histories of displacements of the inner

Fig. 18b, when the amplitude of excitation increases to $\alpha=1.5$, the trajectory hits the discontinuity boundary $I_{0}$ at when the capsule has backward drift (i.e. $v_{2}<0$ ). Identification of both bifurcations are very important for the capsule system, since avoidance of such events may reduce the energy loss caused by external friction, so that improving energy efficiency of the system.

\subsection{Influence of frequency of excitation}

The effect of frequency of excitation $\omega$ on capsule dynamics is studied in this section. Bifurcation diagrams and average progressions of the capsule com- mass (black solid line) and the capsule (red dash line) obtained for $\alpha=0.10,0.80,1.50,3.12,3.37,5.43$ and 6.50. Coexisting attractors are indicated by red dots. (Color figure online)

puted for $\kappa_{2}=0,5$, and 20 are presented, respectively, in Figs. 19, 20 and 21 with additional panels illustrating phase trajectories and time histories of displacements of the inner mass and the capsule. In general, when the frequency of excitation is low $(\omega<0.5)$, the phase trajectories of the capsule system are twisted and several impacts are encountered as illustrated in Figs. 19c, d, 20c, d and 21c, d. When the frequency is sufficiently large $(\omega>1.5)$, the dynamic responses of the capsule are mainly period-1 motions. Comparing the bifurcation diagrams in Figs. 19a, 20a, and $21 \mathrm{a}$, one can see that, the response of the capsule for $\kappa_{2}=0$ is mainly period- 1 motion, and an immediate change of capsule direction due to grazing contact is recorded. As $\kappa_{2}$ increases, the dynamics 


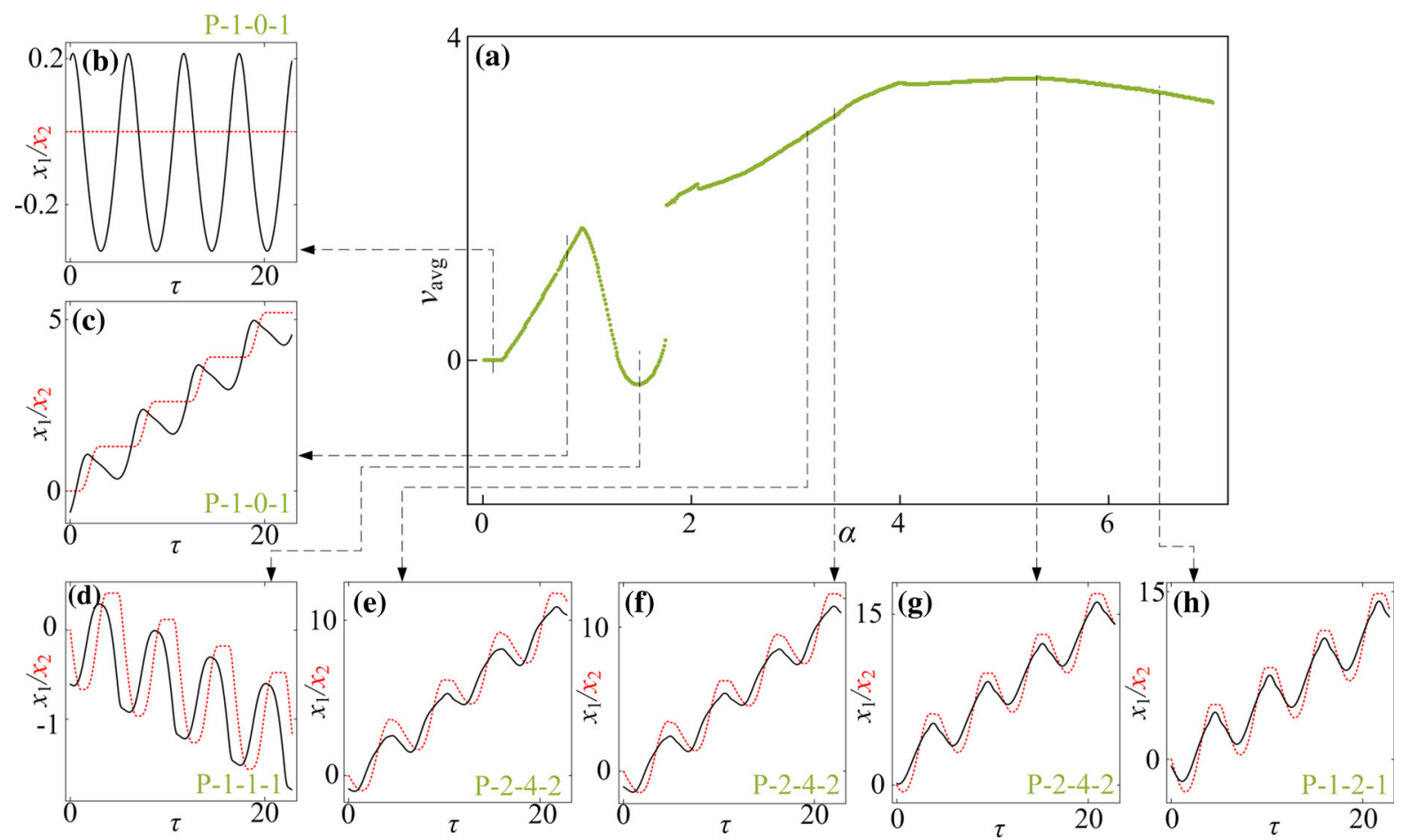

Fig. 16 a Average progression velocities under variation of amplitude of excitation $\alpha$ calculated for $\omega=1.1, \zeta=0.05, \gamma=$ $1, \delta_{1}=0.02, \delta_{2}=1.0, \kappa_{1}=2, \kappa_{2}=20$. b-h Additional windows demonstrate the time histories of displacements of the inner

of the capsule system becomes complex. For example, when $\kappa_{2}=5$, a number of grazing bifurcations, periodic doublings, and reverse period doublings are recorded. These grazing singularities induce sudden jumps of average progression velocities of the capsule at $\omega=0.384,0.585,1.036,1.170,1.197$, and 1.244 , and the jump at $\omega=0.1169$ yields the fastest progression velocity for $\kappa_{2}=5$, which is shown in Fig. 20i. In addition, the period doubling leads to period-2 motion for $\omega \in[0.525,0.561]$, [0.616, 0.687] and $[0.834,0.900]$. Phase trajectories of three selected periodic-2 responses within these parameter regions are plotted in Fig. 20e-g. When $\kappa_{2}=20$, the bifurcation pattern shown in Fig. 21a becomes more complex. Two blow-up windows were plotted to show more details of these bifurcations. The first window shows the switches between period-1 and chaotic motions through several grazing contacts, while the second one illustrates the successive reverse period doublings from chaotic to period-1 response.

Comparing the average velocities of the capsules with different $\kappa_{2}$, it can be found that, when the fre- mass (black solid line) and the capsule (red dash line) obtained for $\alpha=0.10,0.80,1.50,3.12,3.37,5.34$ and 6.50. (Color figure online)

quency of excitation is low $(\omega<0.5)$, any small variations of excitation frequency may affect the average speed significantly. As can be seen from Fig. 19b, two local peaks are recorded at $\omega=0.479$ and 0.986 for a P-1-2-3 and a P-1-2-1 motion, respectively. The maximal average velocity for $\kappa_{2}=5$ is achieved by a P-1-1-1 motion at $\omega=1.169$. For $\kappa_{2}=20$, the maximal velocity is achieved by a P-1-1-2 motion at $\omega=1.226$. Comparing these two motions presented in Figs. 20o and 211, both inner masses have two phases of forward motion in each period of excitation, and the backward motions of both capsules are stopped by the second forward phase of the inner mass. As a result, the progression velocities for both capsules are improved.

\section{Energy consumption and cabin length}

In this section, we will study the best control parameters for capsule progression. In Fig. 15, the fastest speed of the capsule is achieved by the system with two-sided constraints $\left(\kappa_{2}=5\right)$. Therefore, as shown in Fig. 22c, 
Fig. 17 Trajectories on the phase plane

$\left(x_{1}-x_{2}, v_{1}-v_{2}\right)$ and time histories of velocities of the capsule, $v_{2}$ obtained for $\omega=1.1, \zeta=0.05, \gamma=$ $1, \delta_{1}=0.02, \delta_{2}=$

$1.0, \kappa_{1}=2, \kappa_{2}=20: \mathbf{a}$ $\alpha=0.91$ (before grazing) and $\mathbf{b} \alpha=0.95$ (after grazing), where

$I_{0}:=\left(x_{1}-x_{2}\right)-\delta_{1}=0$ (red lines),

$I_{1}:=\left(x_{1}-x_{2}\right)+\delta_{2}=0$ (blue lines), and

$I_{2}:=\left(x_{1}-x_{2}\right)+2 \zeta\left(v_{1}-\right.$ $\left.v_{2}\right)+\kappa_{2}\left(x_{1}-x_{2}+\delta_{2}\right)+1=0$ (green lines). (Color figure online)
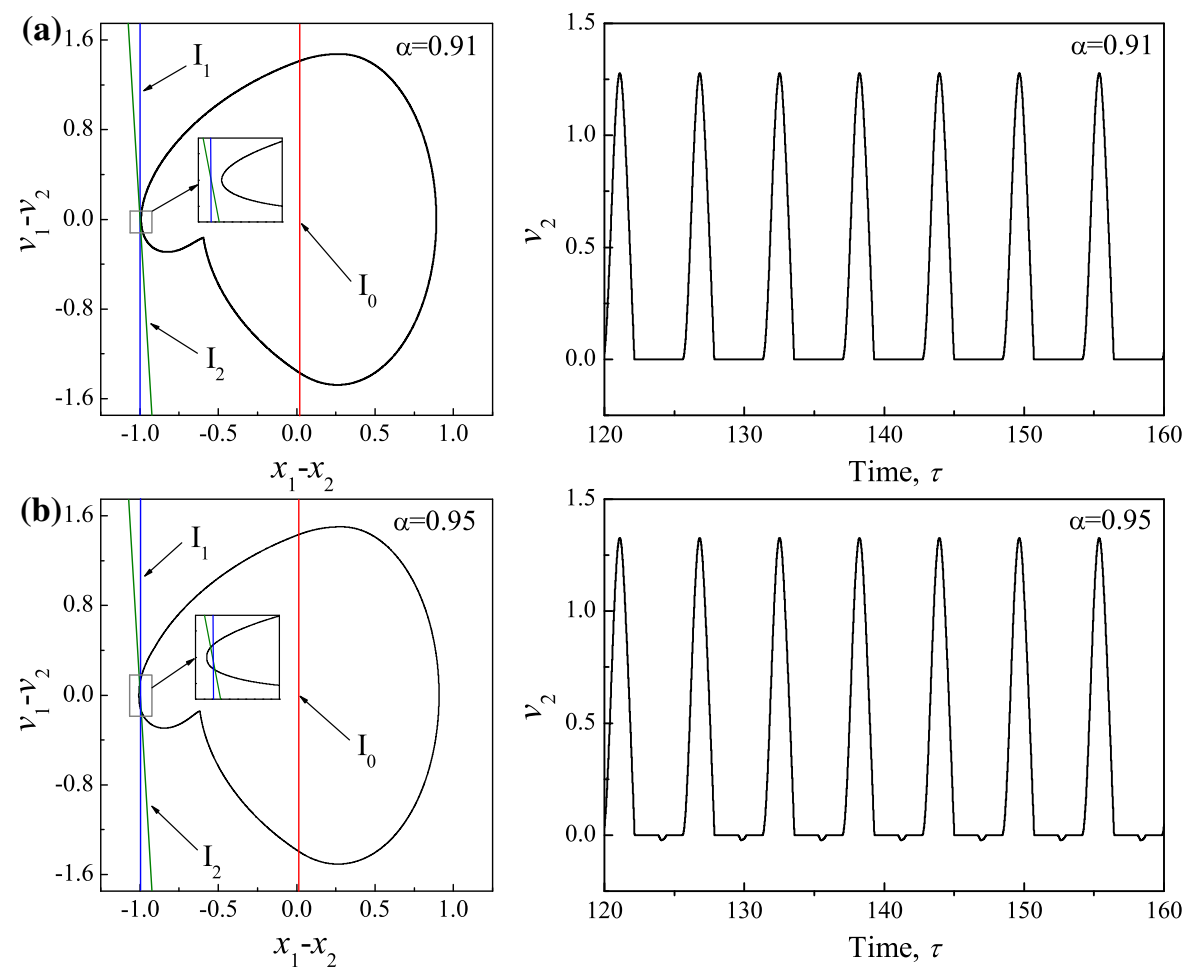

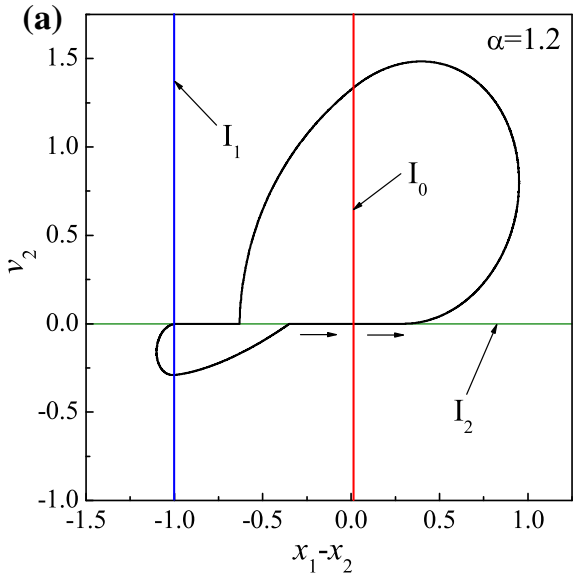

Fig. 18 Trajectories on the phase plane $\left(x_{1}-x_{2}, v_{2}\right)$ computed for $\omega=1.1, \zeta=0.05, \gamma=1, \delta_{1}=0.02, \delta_{2}=1.0, \kappa_{1}=$ $2, \kappa_{2}=5: \mathbf{a} \alpha=1.2$ (before the boundary-intersection crossing bifurcation) and $\mathbf{b} \alpha=1.5$ (after the boundary-intersection

$\mathrm{f}$, an optimum approach to control capsule progression is to increase the amplitude of excitation as large as possible. However, larger amplitude consumes more energy, so that the control parameters for the fastest

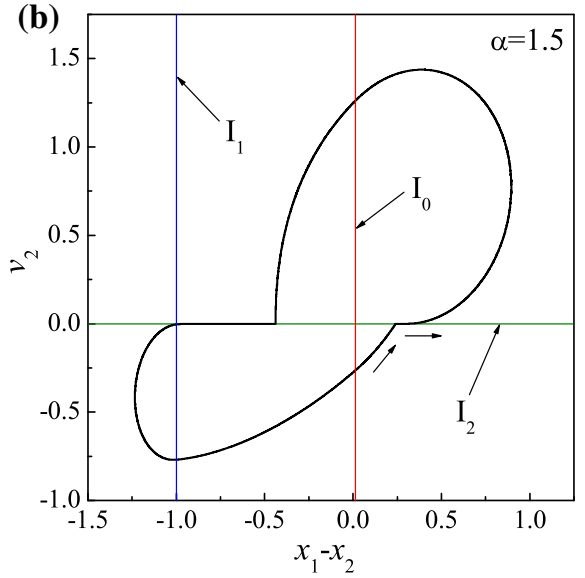

crossing bifurcation), where $I_{0}:=\left(x_{1}-x_{2}\right)-\delta_{1}=0$ (red lines), $I_{1}:=\left(x_{1}-x_{2}\right)+\delta_{2}=0$ (blue lines), and $I_{2}:=v_{2}=0$ (green lines). (Color figure online)

progression are not the most efficient ones [8]. In order to consider this, we introduce the normalised average velocity of the capsule $v_{\mathrm{E}}$, which is given as 

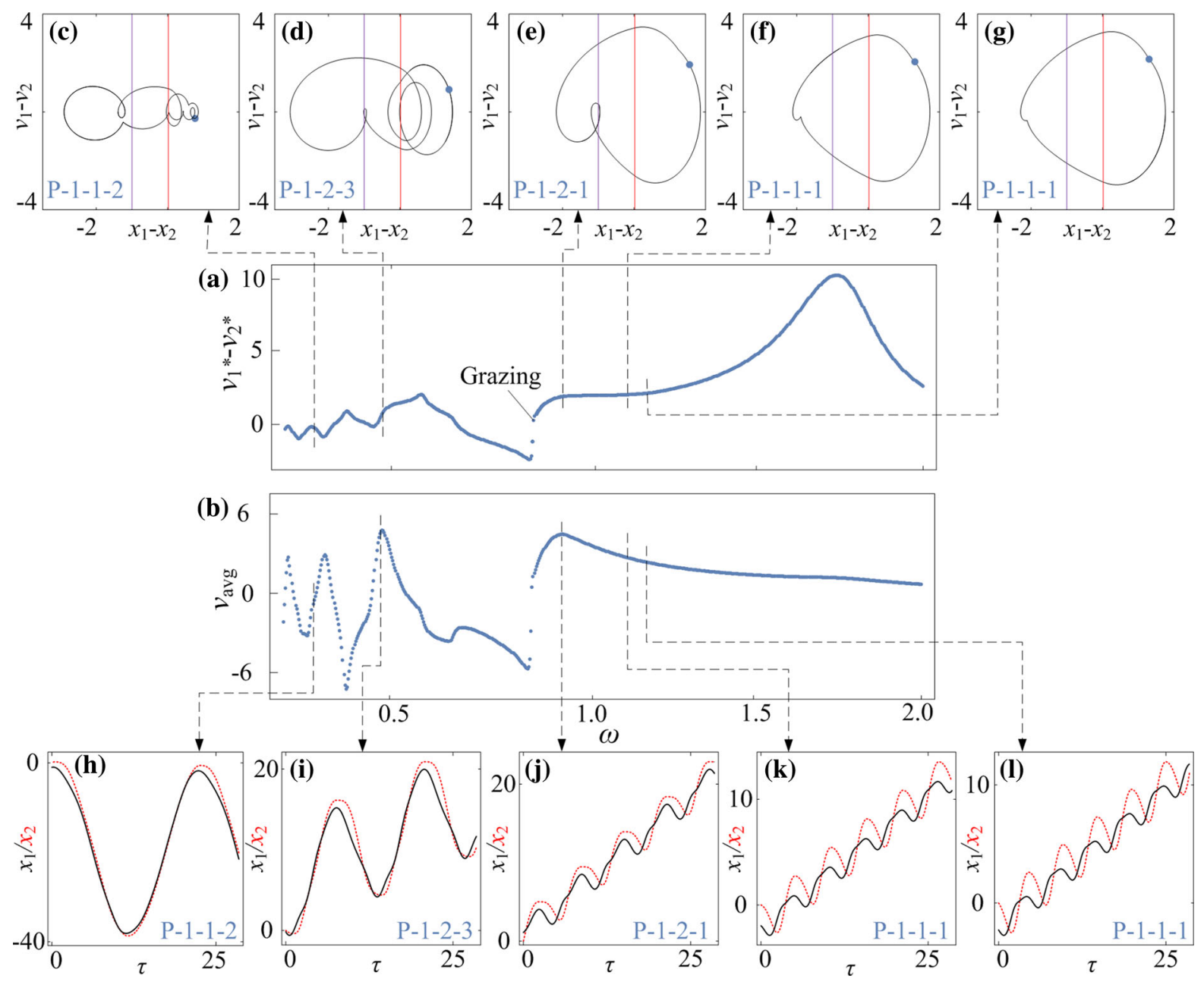

Fig. 19 a Bifurcation diagram and $\mathbf{b}$ average progression velocities of the capsule system under variation of frequency of excitation $\omega$ calculated for $\alpha=3.37, \zeta=0.05, \gamma=1, \delta_{1}=$ $0.02, \delta_{2}=1.00, \kappa_{1}=2, \kappa_{2}=0$. $\mathbf{c}-\mathbf{g}$ Trajectories of the system displayed on the phase plane $\left(x_{1}-x_{2}, v_{1}-v_{2}\right)$, and $\mathbf{h}-\mathbf{l}$ time histories of displacements of the inner mass (black

$v_{\mathrm{E}}=\frac{N v_{\mathrm{avg}}}{\int_{0}^{N T} \alpha \cos (\omega \tau) v_{1}(\tau) \mathrm{d} \tau}$.

Based on Eq. (13), the results in Fig. 22 were recalculated and the new results are shown in Fig. 23. It is seen that the maximal $v_{\mathrm{E}}$ is achieved at $\alpha=0.92$ through a P-1-0-1 motion. In other words, the capsule system with one-sided constraint has the most energy-efficient progression. As the amplitude of excitation $\alpha$ increases, the normalised average velocity $v_{\mathrm{E}}$ drops dramatically.

Another consideration of optimisation is spacesaving for the capsule cabin, i.e. to use the smallest capsule to realise the fastest progression. Thus, our pur- solid lines) and the capsule (red dash line) obtained for $\omega=$ $0.284,0.479,0.986,1.169$, and 1.226. Locations of the left and the right impact surfaces are shown by purple and red lines, respectively. Poincaré sections are marked by blue dots on the phase plane. (Color figure online)

pose is to shorten the cabin length of the capsule system as illustrated in Fig. 24, where the required cabin length is determined by the relative displacement between the inner mass and the capsule, $x_{1}-x_{2}$. Specifically, the shortest cabin length can be calculated as

$L=\max \left(x_{1}-x_{2}\right)-\min \left(x_{1}-x_{2}\right)$,

and we define the relative average progression velocity as 

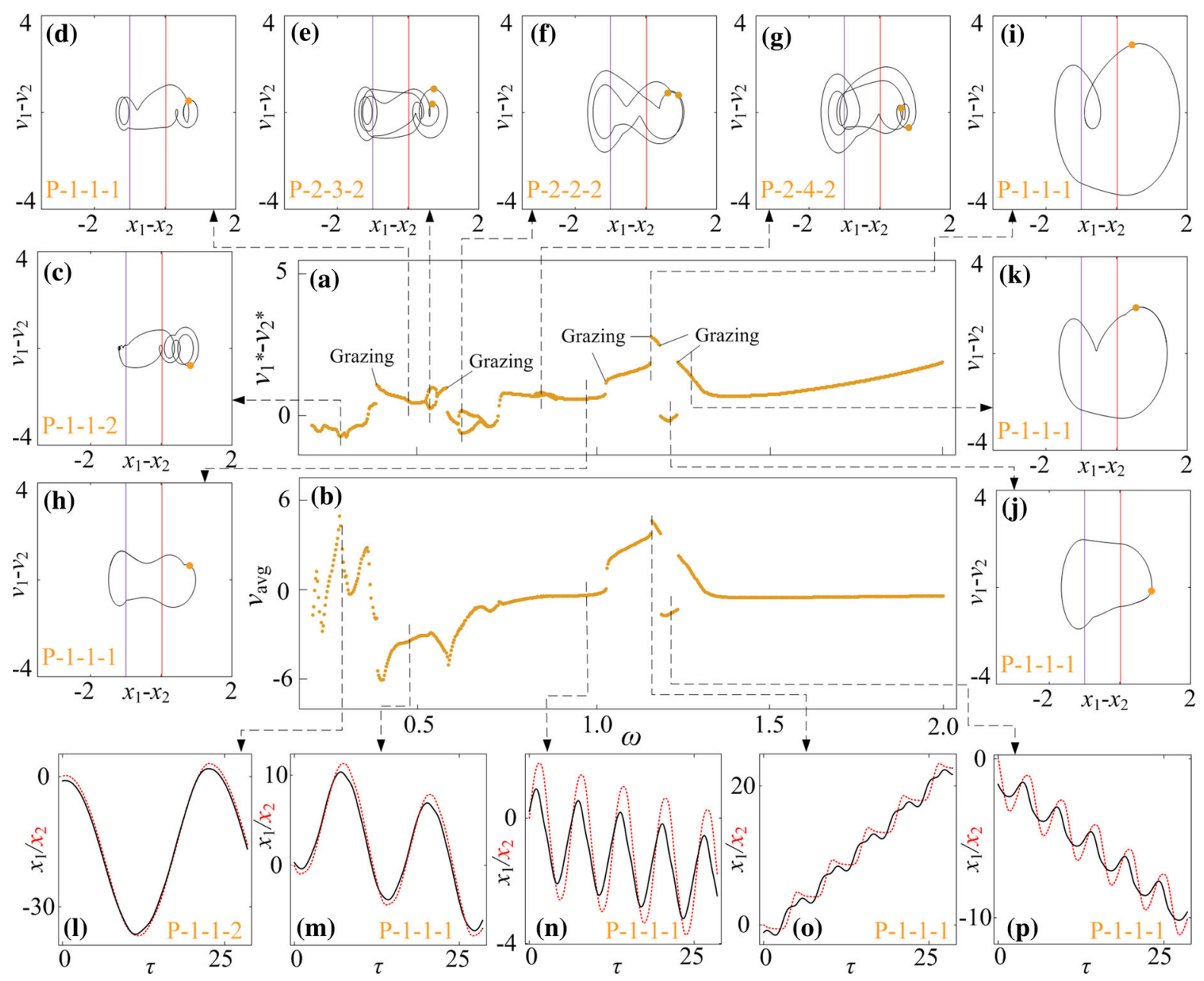

Fig. 20 a Bifurcation diagram and $\mathbf{b}$ average progression velocities of the capsule system under variation of frequency of excitation $\omega$ calculated for $\alpha=3.37, \zeta=0.05, \gamma=1, \delta_{1}=$ $0.02, \delta_{2}=1.00, \kappa_{1}=2, \kappa_{2}=5$. $\mathbf{c}-\mathbf{k}$ Trajectories of the system displayed on the phase plane $\left(x_{1}-x_{2}, v_{1}-v_{2}\right)$ obtained for $\omega=0.284,0.479,0.548,0.629,0.854,0.986,1.169,1.226$,

$v_{\mathrm{L}}=\frac{v_{\mathrm{avg}}}{L}$.

The calculations of the relative average progression velocity were carried out, and the results are presented in Fig. 25. As can be seen from the figure, the optimum progression for the minimal requirement of cabin length is obtained at $\alpha=3.98$, where a P-1-2-1 motion is recorded. Comparing the capsules with different left springs, the system with a strong left spring $\left(\kappa_{2}=20\right)$ has the largest velocity and the minimal requirement of cabin length as demonstrated in Fig. 25f, g.

and 1.244. Locations of the left and the right impact surfaces are shown by purple and red lines, respectively. l-p Time histories of displacements of the inner mass (black solid lines) and the capsule (red dash line) obtained for $\omega=0.284,0.479,0.986,1.169$, and 1.226. Poincaré sections are marked by orange dots on the phase plane. (Color figure online)

\section{Concluding remarks}

Vibro-impact dynamics of the capsule systems with one-sided and two-sided constraints were studied in this paper. Our concern focused on optimising the control parameters of these system, i.e. mass ratio, stiffness ratios, gaps of contact, frequency and amplitude of excitation, in terms of average progression velocity, energy consumption, and cabin length. Bifurcation analysis was conducted by monitoring the relative velocity between the inner mass and the capsule 

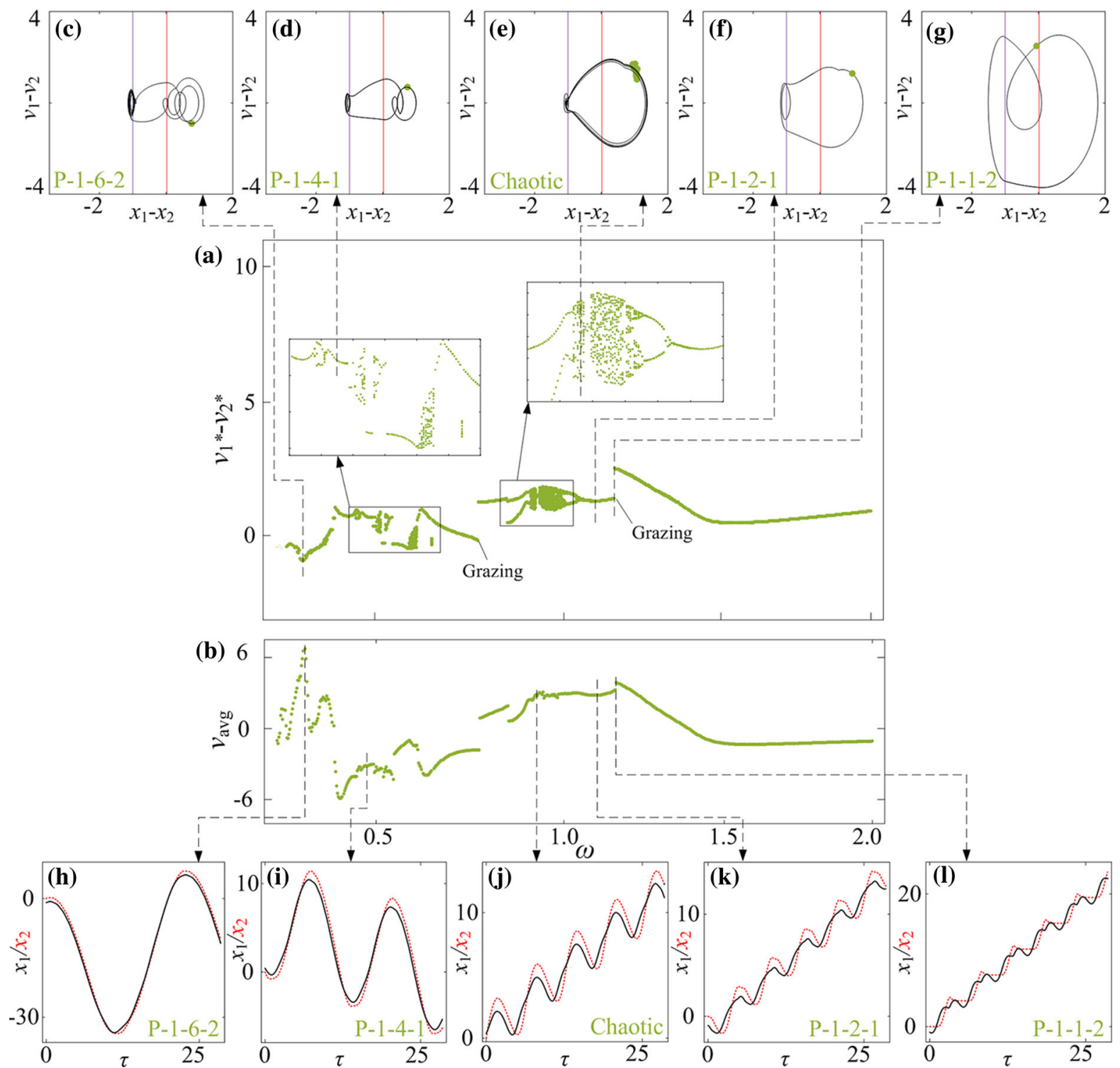

Fig. 21 a Bifurcation diagram and $\mathbf{b}$ average progression velocities of the capsule under variation of frequency of excitation $\omega$ calculated for $\alpha=3.37, \zeta=0.05, \gamma=1, \delta_{1}=0.02, \delta_{2}=$ $1.00, \kappa_{1}=2, \kappa_{2}=20$. $\mathbf{c}-\mathbf{g}$ Trajectories of the system displayed on the phase plane $\left(x_{1}-x_{2}, v_{1}-v_{2}\right)$, and $\mathbf{h}-\mathbf{l}$ time histories of

and the average progression of the capsule per period of external excitation. Extensive comparative studies were undertaken for three different capsule systems, i.e. the capsule with a right constraint, the capsule with a right and a weak left constraints, and the capsule with a right and a strong left constraints. Our bifurcation studies revealed that the behaviour of the capsule with one-sided constraint was mainly periodic, and its average velocity was always faster than the capsule with two-sided constraints. The dynamic responses of the capsule with two-sided constraints may become very complex when the stiffness of the left spring increases.

For the investigation of influence of mass ratio $\gamma$, it was found that for the considered set of parameters, 

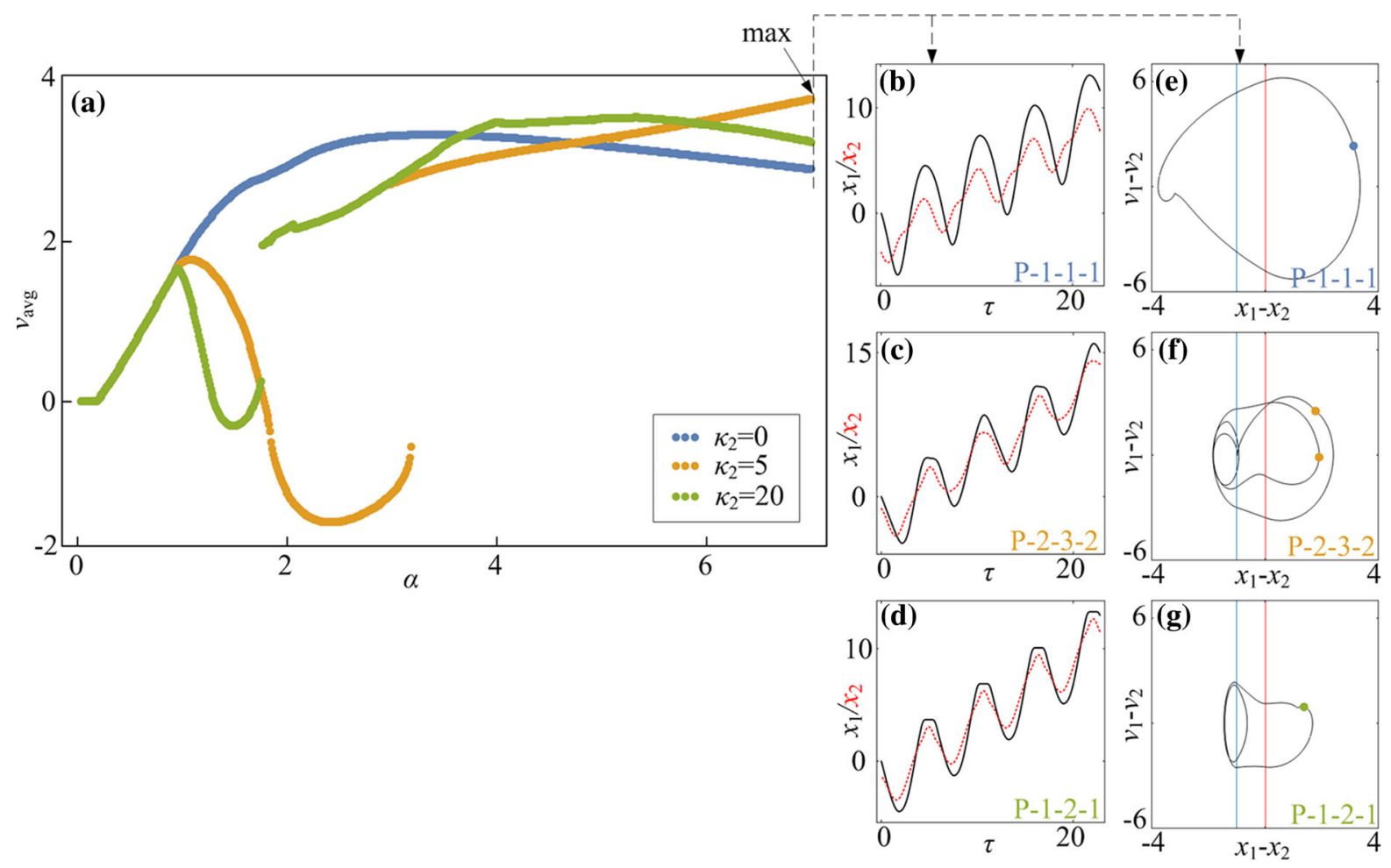

Fig. 22 a Average progression velocities of the capsule systems with $\kappa_{2}=0$ (blue dots), $\kappa_{2}=5$ (orange dots), and $\kappa_{2}=20$ (green dots) are plotted as functions of amplitude of excitation $\alpha$ calculated for $\omega=1.1, \zeta=0.05, \gamma=1, \delta_{1}=0.02, \delta_{2}=1.0$, and $\kappa_{1}=2$. Additional windows demonstrate the time histories of displacements of the inner mass (black solid line) and the cap-

the system experienced period-1 motion for all the studied values of mass ratio, and the direction of capsule progression can be altered through the grazing contact between the inner mass and the right constraint. As the mass ratio increases, vibro-impact motion of the inner mass becomes ineffective so that average progression of the capsule decreases. For the scenario of the capsule with two-sided symmetrical constraints, i.e. $\delta_{1}=\delta_{2}$ and $\kappa_{1}=\kappa_{2}$, the capsule cannot progress for any values of the mass ratio. By investigating the influence of stiffness ratios $\kappa_{1}$ and $\kappa_{2}$, our studies indicated that the maximal average speed can be achieved by the capsule with one-sided constraint, and introduction of the second constraint with any values of stiffness may reduce the average speed of the capsule. Based on the bifurcation study of amplitude of excitation, it was found that the capsule with a right and a strong left constraints had more complex responses than the other two capsules. sule (red dash line) obtained for $\mathbf{b} \kappa_{2}=0$, c 5, d 20, and the trajectories on the phase plane $\left(x_{1}-x_{2}, v_{1}-v_{2}\right)$ obtained for e $\kappa_{2}=0, \mathbf{f} 5, \mathbf{g} 20$. Locations of the left and the right impact surfaces are shown by blue and red lines, respectively. Poincaré sections are marked by blue, orange, and green dots on the phase plane. (Color figure online)

Furthermore, the capsule with a right and a weak left constraints could move faster than the others providing that a sufficiently large amplitude of excitation is applied. Our investigation on the frequency of excitation shows that, when the frequency is low $(\omega<0.5)$, the dynamic responses of the capsule are complex, and any small perturbation on the frequency may results in a significant change of its average velocity. Once the frequency is sufficiently large $(\omega>1.5)$, the dynamic responses of the system are mainly period-1 motions, and the grazing contact with the left constraint could help to enhance the progression speed of the capsule.

In addition, influence of gap of contact for the left constraint was studied. We have conducted the investigation for both positive and negative gaps, which the later one represents a prestressed internal mass. Our investigation suggests that if the miniaturization 


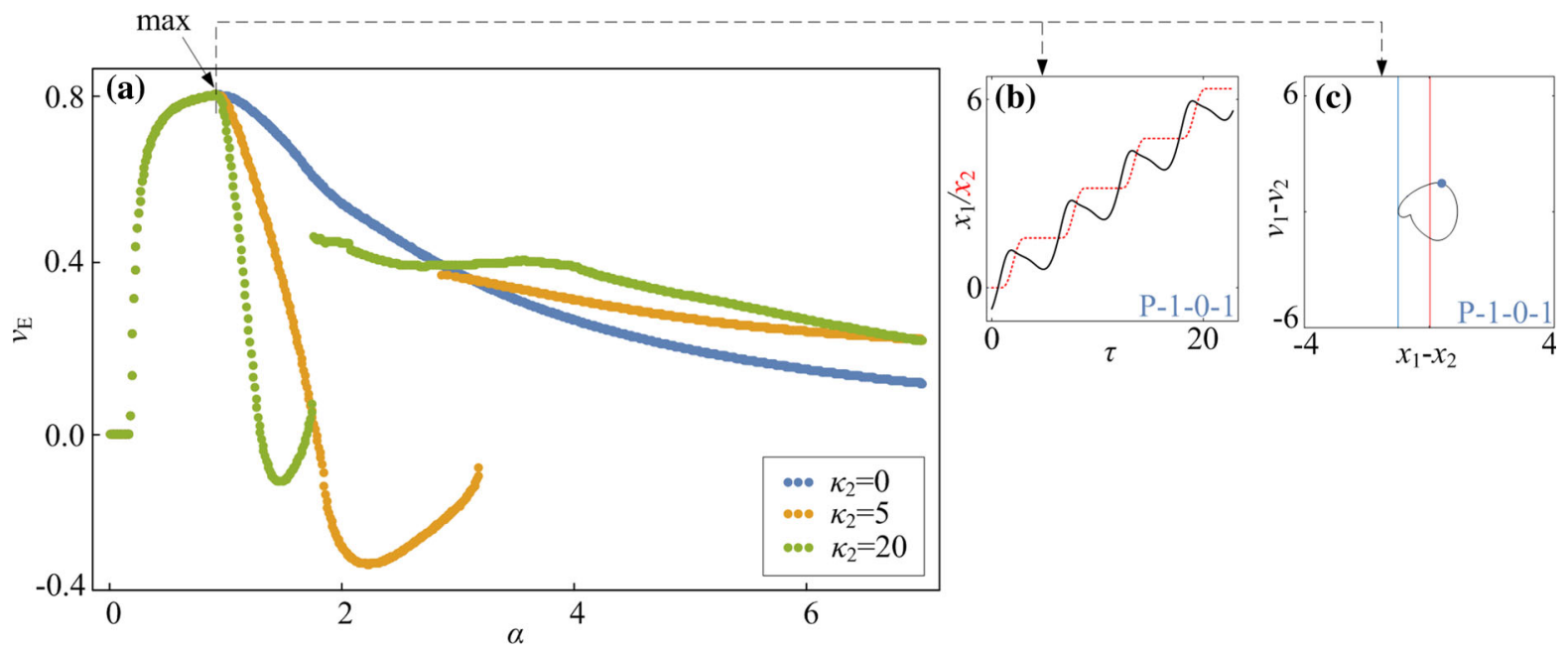

Fig. 23 a Average progression velocities per energy consumption with $\kappa_{2}=0$ (blue dots), $\kappa_{2}=5$ (orange dots), and $\kappa_{2}=20$ (green dots) are plotted as functions of amplitude of excitation $\alpha$ calculated for $\omega=1.1, \zeta=0.05, \gamma=1, \delta_{1}=0.02, \delta_{2}=1.0$, and $\kappa_{1}=2$. Additional windows demonstrate $\mathbf{b}$ the time histories of displacements of the inner mass (black solid line) and the

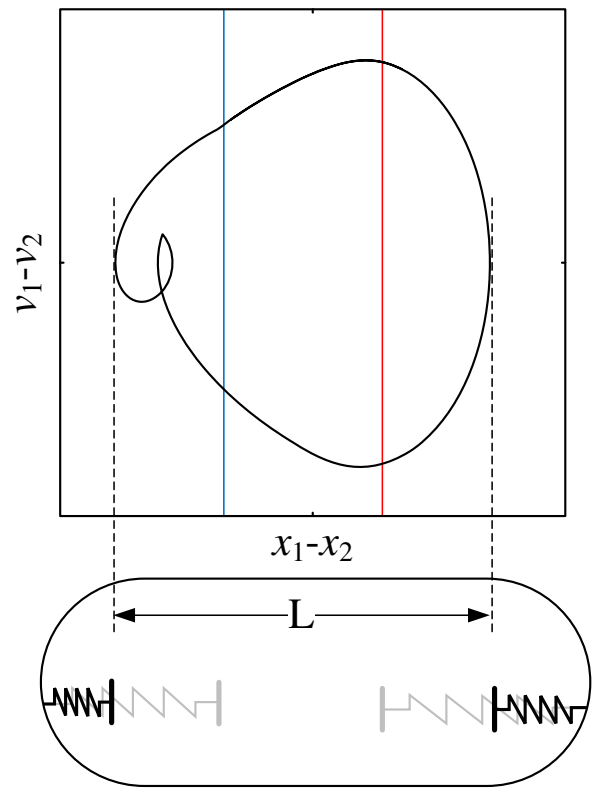

Fig. 24 Required cabin length of the capsule which is determined by the relative displacement between the inner mass and the capsule, $x_{1}-x_{2}$. Locations of the left and the right impact surfaces are shown by blue and red lines, respectively. (Color figure online)

of the capsule is required, the prestressed constraint using a weak spring could be a viable option for prototype design, although the introduction of the left capsule (red dash line) and $\mathbf{c}$ the trajectories on the phase plane $\left(x_{1}-x_{2}, v_{1}-v_{2}\right)$ obtained for $\alpha=0.92$, where the maximal $v_{\mathrm{E}}$ is achieved. Locations of the left and the right impact surfaces are shown by blue and red lines, respectively. Poincaré sections are marked by blue dots on the phase plane. (Color figure online)

spring may affect the average speed of the capsule. We have also observed two important bifurcations which are the grazing bifurcation for the transition to backward drift and the boundary-intersection crossing bifurcation. They are important to be identified, since avoidance of such bifurcations could significantly reduce the energy loss caused by external friction so that improving energy efficiency of the entire capsule system.

Finally, calculations regarding to energy efficiency and cabin length were carried out. Our calculated results revealed that the capsule with a right constraint was the most energy-efficient, and the capsule with a right and a strong left constraints required the minimal cabin length. Based on the analyses above, our strategy for optimisation can be summarised as follows. When capsule speed is paramount, one can employ the two-sided capsule with a weak left constraint and apply a large amplitude of excitation. When energy consumption is taken into account, the one-sided capsule is preferable. When a miniaturized capsule prototype is needed, the two-sided capsule with a strong left constraint is the best choice for prototyping.

In conclusion, our motivation to investigate various design aspects of the vibro-impact capsule system 

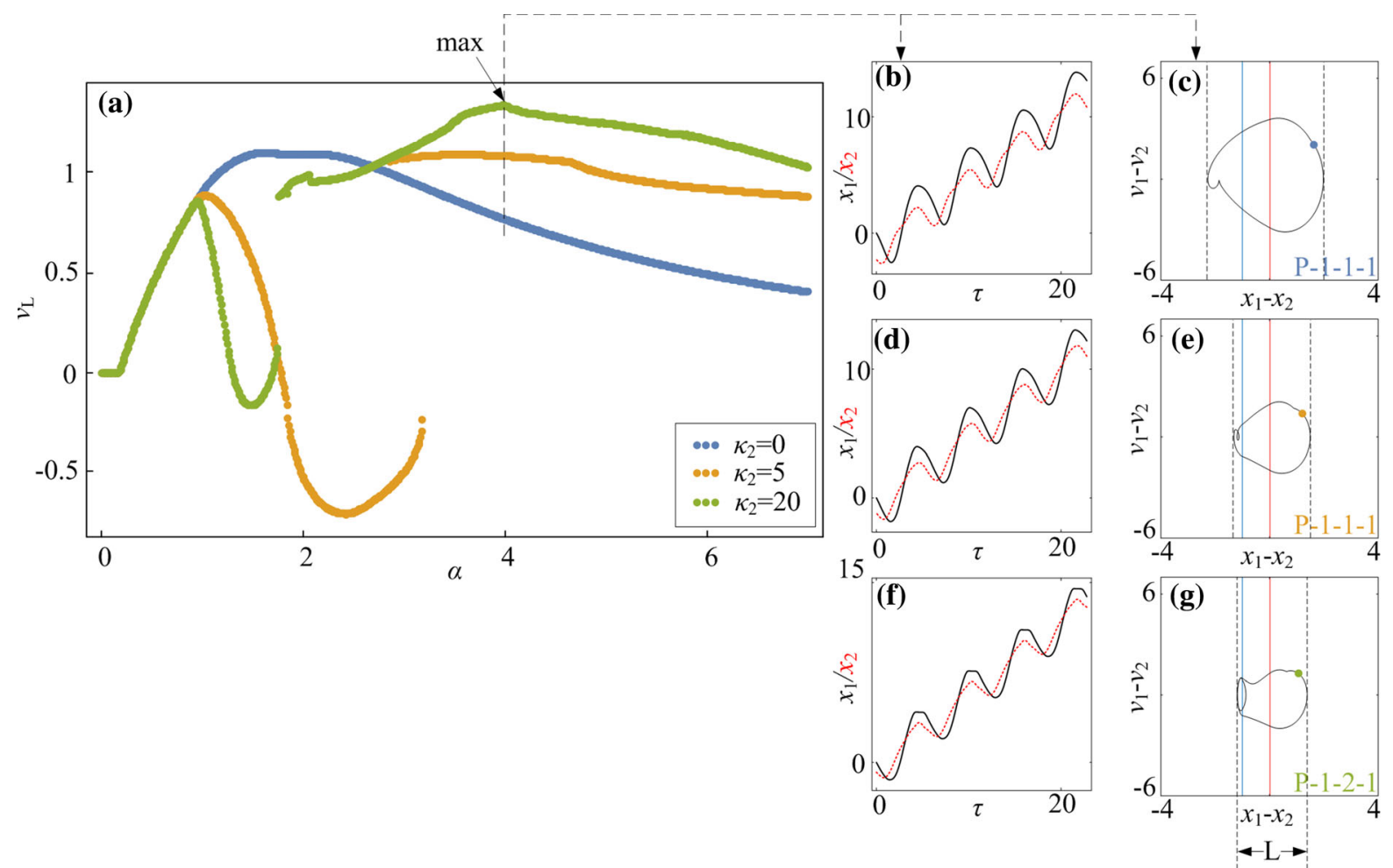

Fig. 25 Relative average progression velocities with $\kappa_{2}=0$ (blue dots), $\kappa_{2}=5$ (orange dots), and $\kappa_{2}=20$ (green dots) are plotted as functions of amplitude of excitation $\alpha$ calculated for $\omega=1.1, \zeta=0.05, \gamma=1, \delta_{1}=0.02, \delta_{2}=1.0$, and $\kappa_{1}=2$. Additional windows demonstrate the time histories of displacements of the inner mass (black line) and the capsule (red line) and the trajectories on the phase plane $\left(x_{1}-x_{2}, v_{1}-v_{2}\right)$ obtained

for pipeline inspection was achieved by taking a comparative study on the capsule systems with one-sided and two-sided constraints. Optimum design parameters (e.g. stiffness ratios, mass ratio) and control parameters (e.g. frequency and amplitude of excitation) were suggested for prototype design with regards to capsule speed, energy efficiency, and capsule dimension, which are the main contribution of this paper. Future works will focus on implementation of the capsule prototype and its experimental testing in a fluid pipeline. It is also worth to develop a general control method for such type of nonlinear systems, e.g. [31,32], particularly when the number of nonlinearities increases, the dynamics of the system will become more complex from periodic motion to chaos.

Acknowledgements Dr. Yang Liu would like to acknowledge the financial support from EPSRC for his First Grant (Grant No. EP/P023983/1). Dr. Yao Yan was supported by the National for $\alpha=3.98, \mathbf{b}, \mathbf{c} \kappa_{2}=0$, d, e $\kappa_{2}=5$, and $\mathbf{f}, \mathbf{g} \kappa_{2}=20$, where the minimal requirement of cabin length $v_{\mathrm{L}}$ is recorded. Locations of the left and the right impact surfaces are shown by blue and red lines, respectively. Poincaré sections are marked by blue, orange, and green dots on the phase plane. (Color figure online)

Natural Science Foundation of China (Grant No. 11572224 and 11502048) and the Fundamental Research Funds for the Central Universities (Grant No. ZYGX2015KYQD033).

Open Access This article is distributed under the terms of the Creative Commons Attribution 4.0 International License (http://creativecommons.org/licenses/by/4.0/), which permits unrestricted use, distribution, and reproduction in any medium, provided you give appropriate credit to the original author(s) and the source, provide a link to the Creative Commons license, and indicate if changes were made.

\section{References}

1. Shukla, A., Karki, H.: Application of robotics in onshore oil and gas industry - a review part i. Robot. Auton. Syst. 75, 490-507 (2016)

2. Shukla, A., Karki, H.: Application of robotics in onshore oil and gas industry-a review part ii. Robot. Auton. Syst. 75, 508-524 (2016) 
3. Tiratsoo, J.: Pipeline Pigging Technology ( $2^{\text {nd }}$ Edition). Gulf Professional Publishing, Houston (1992)

4. Chernousko, F.L.: The optimum rectilinear motion of a twomass system. J. Appl. Math. Mech. 66, 1-7 (2002)

5. Bolotnik, N.N., Zeidis, I.M., Zimmermann, K., Yatsun, S.F.: Dynamics of controlled motion of vibration-driven systems. J. Comput. Syst. Sci. Int. 45, 157-167 (2006)

6. Fang, H.B., Xu, J.: Dynamic analysis and optimization of a three-phase control mode of a mobile system with an internal mass. J. Vib. Control 17, 19-26 (2011)

7. Nagy, Z., Leine, R.I., Frutiger, D.R., Glocker, C., Nelson, B.J.: Modeling the motion of microrobots on surfaces using nonsmooth multibody dynamics. IEEE Trans. Robot. 28, 1058-1068 (2012)

8. Liu, Y., Wiercigroch, M., Pavlovskaia, E., Yu, H.: Modelling of a vibro-impact capsule system. Int. J. Mech. Sci. 66, 2-11 (2013)

9. Liu, Y., Pavlovskaia, E., Wiercigroch, M.: Vibro-impact responses of capsule system with various friction models. Int. J. Mech. Sci. 72, 39-54 (2013)

10. Liu, Y., Wiercigroch, M., Pavlovskaia, E., Peng, Z.K.: Forward and backward motion control of a vibro-impact capsule system. Int. J. Non-Linear Mech. 70, 30-46 (2015)

11. Fang, H.B., Xu, J.: Dynamics of a mobile system with an internal acceleration-controlled mass in a resistive medium. J. Sound Vib. 330, 4002-4018 (2011)

12. Fang, H.B., Xu, J.: Dynamics of a three-module vibrationdriven system with non-symmetric coulomb's dry friction. Multibody Syst. Dyn. 27, 455-485 (2012)

13. Zhan, X., Xu, J.: Locomotion analysis of a vibration-driven system with three acceleration-controlled internal masses. Adv. Mech. Eng. 7, 1-12 (2015)

14. Chernousko, F.: Two-dimensional motions of a body containing internal moving masses. Meccanica 51, 3203-3209 (2016)

15. Zhan, X., Xu, J., Fang, H.: Planar locomotion of a vibrationdriven system with two internal masses. Appl. Math. Model. 40, 871-885 (2016)

16. Vetchanin, E.V., Mamaev, I.S., Tenenev, V.A.: The selfpropulsion of a body with moving internal masses in a viscous fluid. Regul. Chaot. Dyn. 18, 100-117 (2013)

17. Liu, Y., Pavlovskaia, E., Wiercigroch, M.: Experimental verification of the vibro-impact capsule model. Nonlinear Dyn. 83, 1029-1041 (2016)

18. Liu, Y., Islam, S., Pavlovskaia, E., Wiercigroch, M.: Optimization of the vibro-impact capsule system, Strojniš ki vestnik. J. Mech. Eng. 62, 430-439 (2016)

19. Páez Cháez, J., Liu, Y., Pavlovskaia, E., Wiercigroch, M.: Path-following analysis of the dynamical response of a piecewise-linear capsule system. Commun. Nonlinear Sci. Numer. Simul. 37, 102-114 (2016)
20. Liu, Y., Páez Cháez, J.: Controlling multistability in a vibro-impact capsule system. Nonlinear Dyn 88, 1289-1304 (2017)

21. Gutiérrez, E., Arrowsmith, D.K.: Control of a double impacting mechanical oscillator using displacement feedback. Int. J. Bifurc. Chaos 14, 3095-3113 (2004)

22. Lee, J.Y., Yan, J.J.: Position control of double-side impact oscillator. Mech. Syst. Signal Process. 21, 1076-1083 (2007)

23. Luo, G.W., Lv, X.H., Shi, Y.Q.: Vibro-impact dynamics of a two-degree-of-freedom periodically-forced system with a clearance: diversity and parameter matching of periodicimpact motions. Int. J. Non-Linear Mech. 65, 173-195 (2014)

24. Yang, G., Xu, W., Gu, X., Huang, D.: Response analysis for a vibroimpact duffing system with bilateral barriers under external and parametric gaussian white noises. Chaos Solitons Fract. 87, 125-135 (2016)

25. Kumar, P., Narayanan, S., Gupta, S.: Bifurcation analysis of a stochastically excited vibro-impact duffing-van der pol oscillator with bilateral rigid barriers. Int. J. Mech. Sci. (2016). doi:10.1016/j.ijmecsci.2016.12.009

26. Andreaus, U., Angelis, M.D.: Nonlinear dynamic response of a base-excited SDOF oscillator with double-side unilateral constraints. Nonlinear Dyn. 84, 1447-1467 (2016)

27. Hao, Z., Cao, Q., Wiercigroch, M.: Two-sided damping constraint control strategy for high-performance vibration isolation and end-stop impact protection. Nonlinear Dyn. 86, 2129-2144 (2016)

28. Ing, J.: Near grazing dynamics of piecewise linear oscillators, Ph.D. thesis, School of Engineering, University of Aberdeen, Aberdeen, UK (2008)

29. Yusupov, A., Liu, Y.: Development of a self-propelled capsule robot for pipeline inspection, In: Proceedings of the $22^{\text {nd }}$ International Conference on Automation and Computing, Colchester, UK, pp. 84-88 (2016)

30. Burden, R.L., Faires, D.J.: Numerical Analysis, 9th edn. Brooks/Cole, Boston (2011)

31. Yin, C., Chen, Y., Zhong, S.: Fractional-order sliding mode based extremum seeking control of a class of nonlinear systems. Automatica 50(12), 3173-3181 (2014)

32. Yin, C., Cheng, Y., Chen, Y., Stark, B., Zhong, S.: Adaptive fractional-order switching-type control method design for 3D fractional-order nonlinear systems. Nonlinear Dyn. 82(1), 39-52 (2015) 